\title{
Exact solution of the planar motion of three arbitrary point vortices
}

\author{
R. Conte and L. de Seze
}

Service de physique du solide et de résonance magnétique, CEN Saclay, France. DPhG/PSRM/1697/80

\begin{abstract}
We give an exact quantitative solution for the motion of three vortices of any strength, which Poincaré showed to be integrable. The absolute motion of one vortex is generally biperiodic: in uniformly rotating axes, the motion is periodic. There are two kinds of relative equilibrium configuration: two equilateral triangles and one or three colinear configurations, their stability conditions split the strengths space into three domains in which the sets of trajectories are topologically distinct.

According to the values of the strengths and the initial positions, all possible motions are classified. Two sets of strengths lead to generic motions other than biperiodic. First, when the angular momentum vanishes, besides the biperiodic regime there exists an expansion spiral motion and even a triple collision in a finite time, but the latter motion is nongeneric. Second, when two strengths are opposite, the system also exhibits the elastic diffusion of a vortex doublet by the third vortex.

For given values of the invariants, the volume of the phase space of this Hamiltonian system is proportional to the period of the reduced motion, a well known result of the theory of adiabatic invariants. We then formally examine the behaviour of the quantities that Onsager defined only for a large number of interacting vortices.
\end{abstract}

\section{Introduction}

Known from a long time, the problem of the motion of a system of point vortices in interaction presents a particular interest for the study of bidimensional turbulence. As predicted by Onsager (1949) the study of the thermodynamics of a large system of vortices shows the possibility of negative 
temperatures and eddy viscosities (see e.g. Lundgren and Pointin 1977). The case of a small number of vortices was studied, long ago, by Lord Kelvin and Mayer (1878). They used experimental methods to obtain some results on the stability of simple geometric configurations. Recently Novikov (1975) pointed out the interest of the three vortex system as the first elementary interaction process in isotropic turbulence kinetics. Using properties of the triangle he was able to solve the problem of three identical vortices.

While this work was under submission, the referees pointed out to us the existence of a paper to appear. In this paper, Hassan Aref (March 1979), extending the work of Novikov with a symmetry-preserving presentation, qualitatively solved the relative motion of three vortices and undertook a classification of the topology of the phase space; however, he gave no indication on the nature of the absolute motion and no quantitative results, except for two special cases of great importance which he solved completely: the direct or exchange scattering when two strengths are equal and opposite to the third one, and the self-similar motion of a triple collision or a triple expansion to infinity when both the inertia momentum and the angular momentum vanish.

In this paper, we present a method which quantitatively gives the absolute motion in all cases of strengths or initial conditions. The main idea is to introduce reduced variables with a very simple geometrical interpretation and whose number matches the number of degrees of freedom of the system. For three vortices, this can be done by defining one complex variable $\zeta$ which characterizes the shape of the triangle. Knowing the motion of $\zeta$, which happens to be periodic, is then sufficient to derive the behaviour of any physically interesting variable. We thus have obtained detailed results, which resort mainly to fluid mechanics and partly to the field of differentiable dynamical systems.

In the first part, we describe the problem, introduce the reduced motion and discuss its advantages and inconvenients. In the second part, we give the general solution: generically, i.e. for arbitrary strengths and initial positions, the absolute motion of any given vortex is the product of a periodic motion of period $T$ by a uniform rotation or, stated in other words, the motion is periodic when referred to uniformly rotating axes centered at the center of vorticity; in the case of a vortex plasma (zero total strength), the uniform rotation is merely replaced by a uniform translation. Due to its frequent occurrence in nature, we give special consideration to the case of two or three equal strengths: in a given domain of initial conditions, vortices with 
equal strengths have the same motion, up to a shift of $\frac{T}{2}$ or $\frac{T}{3}$ in time and a rotation in space.

The third part, following the Smale's method of study of a differentiable dynamical system, is devoted to the finding of the bifurcation set, i.e. the set of values of the invariants for which the nature of the motion changes. This reduces to the finding of the relative equilibria, which are shown to be of two types, the same than those of the three body problem of celestial mechanics: two equilateral triangles and one or three colinear configurations. The number and stability of these relative equilibria are discussed, thus leading to a separation of the strengths space in three principal domains where the sets of orbits are topologically different; an important result is that, whatever be the strengths domain, the phase space is always multiconnected; for strengths on the boundaries of these domains, the motion must be studied separately for it presents special features.

In the fourth part, we study the absolute motion whenever it differs from the general biperiodic case, i.e. when strengths are on the boundaries of their limiting domains or when initial positions are those of a relative equilibrium. At a relative equilibrium the motion is a rigid body rotation. Otherwise two new generic motions are found. First, for strengths such that the angular momentum vanishes, the vortices can go to infinity in a spiral motion with a fixed asymptotic shape of the triangle; there even exists a spiral motion ending in a triple collision in a finite time, but it is nongeneric since it happens only for a zero value of the inertia momentum. Second, when two strengths are opposite, besides the doubly periodic motion an elastic scattering can occur in which a vortex doublet is diffused by the third vortex; if moreover the third vortex has the strength of one of the two others, a third generic motion exists which is an exchange scattering.

The fifth part consists in formally examining the behaviour of the thermodynamical quantities, of course meaningless for this integrable system, that Onsager defined for a large number of vortices; the volume of the phase space is found to be equal to the period of the relative motion; among the features which could be indicative for a large number of vortices is a possible lack of ergodicity due to a multiple connexity of the phase space.

\section{Description of the problem}

We consider three point vortices $M_{j}(j=1,2,3)$ in a plane with strengths $\kappa_{j}$ and given initial positions. Their motion is ruled by the first order 
differential system:

$$
\forall j=1,2,3: 2 \pi i \frac{\mathrm{d} \bar{z}_{j}}{\mathrm{~d} t}=\sum_{\substack{\ell=1 \\ \ell \neq j}}^{3} \frac{\kappa_{\ell}}{z_{j}-z_{\ell}},
$$

where $z=x+i y$ and the bar denotes the complex conjugation. This system is equivalent to the set of Hamilton equations for the Hamiltonian:

$$
H=-\frac{1}{2 \pi} \sum_{\substack{j \\ j}} \sum_{\substack{\ell \\ \ell}} \kappa_{j} \kappa_{\ell} \log \left|z_{j}-z_{\ell}\right|,
$$

in the conjugate variables $\sqrt{\left|\kappa_{j}\right|} x_{j}$ and $\sqrt{\left|\kappa_{j}\right|} \operatorname{sign}\left(\kappa_{j}\right) y_{j}, j=1,2,3$; therefore $H$ is an invariant and remains equal to the energy $E$. The invariance of $H$ under translation and rotation yields two other invariants:

$$
\begin{aligned}
& \text { the impulse } B=\sum_{j} \kappa_{j} z_{j}=X+i Y, \\
& I=\sum_{j} \kappa_{j}\left|z_{j}\right|^{2}
\end{aligned}
$$

which Poincaré called inertia momentum.

In fact we shall use, instead of $I$, the invariant

$$
J=\sum_{\substack{j \\ j<\ell}} \sum_{\ell} \kappa_{j} \kappa_{\ell}\left|z_{j}-z_{\ell}\right|^{2}=\left(\kappa_{1}+\kappa_{2}+\kappa_{3}\right) I-|B|^{2},
$$

which depends only on the relative positions of the vortices. There are $6-4=2$ remaining degrees of freedom and we suppose the problem to be nondegenerate: $\kappa_{1} \kappa_{2} \kappa_{3}\left(z_{2}-z_{3}\right)\left(z_{3}-z_{1}\right)\left(z_{1}-z_{2}\right) \neq 0$.

Among the six Poisson brackets built from the four known integrals of motion $H, J, X, Y$, only one is nonzero:

$$
\{X, Y\}=\sum_{j} \kappa_{j}=K
$$

Since a Hamiltonian system with $2 N$ variables is integrable in the sense of Liouville when it has $N$ independent invariants in involution, the three vortex system $(N=3)$ is therefore integrable (Poincaré, 1893). The purpose of this paper is to integrate it.

Let us remark that this problem is not affected by the adjunction of an external velocity field made of a uniform translation and a uniform rotation, 
since the new equations of motion

$$
2 \pi i \frac{\mathrm{d} \bar{z}_{j}}{\mathrm{~d} t}=\sum_{\ell}^{\prime} \frac{\kappa_{\ell}}{z_{j}-z_{\ell}}+2 \pi i \bar{v}+2 \pi \omega \bar{z}_{j}, \quad \omega \in \mathbb{R}, \quad v \in \mathbb{C}
$$

reduce to the original ones by the change of variables

$$
Z_{j}=\frac{i v}{\omega}+\left(z_{j}-\frac{i v}{\omega}\right) e^{-i \omega t}
$$

\section{The reduced motion}

The main idea is to match the number of variables and the number of degrees of freedom of this system, so as to keep the minimum number of independent variables. For this purpose, let us define a time dependent complex plane $\zeta$ in which two of the three point vortices remain fixed. This can be achieved by the following transformation

$$
z \rightarrow \zeta=\frac{\left(\kappa_{2}+\kappa_{3}\right) z-\left(\kappa_{2} z_{2}+\kappa_{3} z_{3}\right)}{z_{2}-z_{3}},
$$

where 2 and 3 number two vortices whose sum of strengths is nonzero; the affixes of these two vortices become $\kappa_{3}$ and $-\kappa_{2}$ under the transformation.

It is clearly seen from the definition how a reduced point is geometrically deduced from an absolute point. We shall simply note $\zeta$ the transformed of $M_{1}$ :

$$
\zeta=\xi+i \eta=\frac{K z_{1}-B}{z_{2}-z_{3}}
$$

$\zeta$ therefore represents the shape of the triangle, and the inverse transformation is represented by

$$
\begin{gathered}
z_{1}=z_{1}, \quad z_{2}=\frac{\left(\kappa_{3} K-\kappa_{1} \zeta\right) z_{1}+\left(\zeta-\kappa_{3}\right) B}{s \zeta}, \\
z_{3}=\frac{\left(-\kappa_{2} K-\kappa_{1} \zeta\right) z_{1}+\left(\zeta+\kappa_{2}\right) B}{s \zeta}
\end{gathered}
$$

where $s=\kappa_{2}+\kappa_{3} \neq 0$.

The reader will have noticed the main disadvantage of the above definition of two reduced coordinates $\xi, \eta$ : it does not reflect the invariance of the problem under the permutations of the three elements $\left(\kappa_{j}, z_{j}\right)$ and therefore every result we can get may be uneasy to interpret. Nevertheless, the advantages are numerous. First, every physical quantity can systematically be expressed, as we shall soon see, as a function of $\zeta$ and $K z_{1}-B$ only; moreover, since $\zeta$ is invariant under a change of length and $K z_{1}-B$ extensive in 
the lengths, such a physical function will quite generally be the product of a function of $\zeta$ by a function of $K z_{1}-B$ and we are going to see that this uncoupling between intensive and extensive variables will enable us to solve the motion not only qualitatively but also quantitatively. Secondly, unlike Novikov and Aref, we do not have to eliminate some unphysical portions of our $\zeta$ plane (which will be seen to be the initial conditions plane) since every $\zeta$ point describes a physical situation. Thirdly, this reduction leaves only the required number of degrees of freedom.

\section{The solution for the general case}

To obtain the absolute motion we need only determine the evolution of $\zeta$ and $z_{1}$, since we have the parametric representation (5). Provided $K$ and $B$ do not simultaneously vanish the motions of $z_{1}$ and $\zeta$ are ruled by:

$$
\begin{aligned}
2 \pi i \frac{\mathrm{d} \bar{z}_{1}}{\mathrm{~d} t} & =\frac{1}{K z_{1}-B} \frac{s^{2} \zeta(\zeta+d)}{\left(\zeta+\kappa_{2}\right)\left(\zeta-\kappa_{3}\right)} \\
2 \pi i \frac{\mathrm{d} \bar{\zeta}}{\mathrm{d} t} & =\frac{-1}{\left|K z_{1}-B\right|^{2}} \frac{s|\zeta|^{2}\left[\bar{\zeta}\left(\zeta^{2}+d \zeta-Q\right)-s K(\zeta+d)\right]}{\left(\zeta+\kappa_{2}\right)\left(\zeta-\kappa_{3}\right)}
\end{aligned}
$$

where $d=\kappa_{2}-\kappa_{3}$ and $Q=\kappa_{1} \kappa_{2}+\kappa_{2} \kappa_{3}+\kappa_{3} \kappa_{1}$.

We can express the invariants $E$ and $J$ as functions of $z_{1}$ and $\zeta$ :

$$
\begin{gathered}
J=\left|K z_{1}-B\right|^{2} \frac{\kappa_{1}|\zeta|^{2}+\kappa_{2} \kappa_{3} K}{s|\zeta|^{2}} \\
e^{-4 \pi E}=\left|K z_{1}-B\right|^{2 Q}|\zeta|^{-2 Q}\left|\frac{\zeta-\kappa_{3}}{s}\right|^{2 \kappa_{1} \kappa_{2}}\left|\frac{\zeta+\kappa_{2}}{s}\right|^{2 \kappa_{1} \kappa_{3}}
\end{gathered}
$$

expressions where we notice the factorized dependency on $\zeta$ and $z_{1}$.

Unless $Q$ and $J$ simultaneously vanish, at least one of the two above equations expresses $\left|K z_{1}-B\right|$ as a function of $\zeta$ and from (7) we obtain a first order differential system for $\zeta$. For example if $J$ is nonzero the elimination of $z_{1}$ between (7) and (8) gives:

$$
2 \pi i \frac{\mathrm{d} \bar{\zeta}}{\mathrm{d} t}=\frac{-\left(\kappa_{1}|\zeta|^{2}+\kappa_{2} \kappa_{3} K\right)\left[\bar{\zeta}\left(\zeta^{2}+d \zeta-Q\right)-s K(\zeta+d)\right]}{J\left(\zeta+\kappa_{2}\right)\left(\zeta-\kappa_{3}\right)}
$$


There is no need to solve this system since the equation of the reduced trajectory is given by the straightforward elimination of $z_{1}$ between (8) and (9):

$$
\frac{\kappa_{1}|\zeta|^{2}+\kappa_{2} \kappa_{3} K}{s Q}\left|\frac{\zeta-\kappa_{3}}{s}\right|^{-2 \frac{\kappa_{1} \kappa_{2}}{Q}}\left|\frac{\zeta+\kappa_{2}}{s}\right|^{-2 \frac{\kappa_{1} \kappa_{3}}{Q}}=\frac{J}{Q} e^{4 \pi \frac{E}{Q}}
$$

This represents in the $\zeta$ plane a set of closed orbits, indexed by the nondimensional variable $c=\frac{J}{Q} e^{\frac{4 \pi E}{Q}}$ which is invariant under a change of length or a change of unit of vorticity.

The set of orbits is symmetrical relative to the $\xi$ axis and also, when $\kappa_{2}$ equals $\kappa_{3}$, to the $\eta$ axis. When two vortices are close to each other, they remain as such and therefore the $\zeta$ curves are near to circles in the vicinity of $-\kappa_{2}, \kappa_{3}$ and $\infty$. Figures 2 to 3 show examples of the $\zeta$ plane.

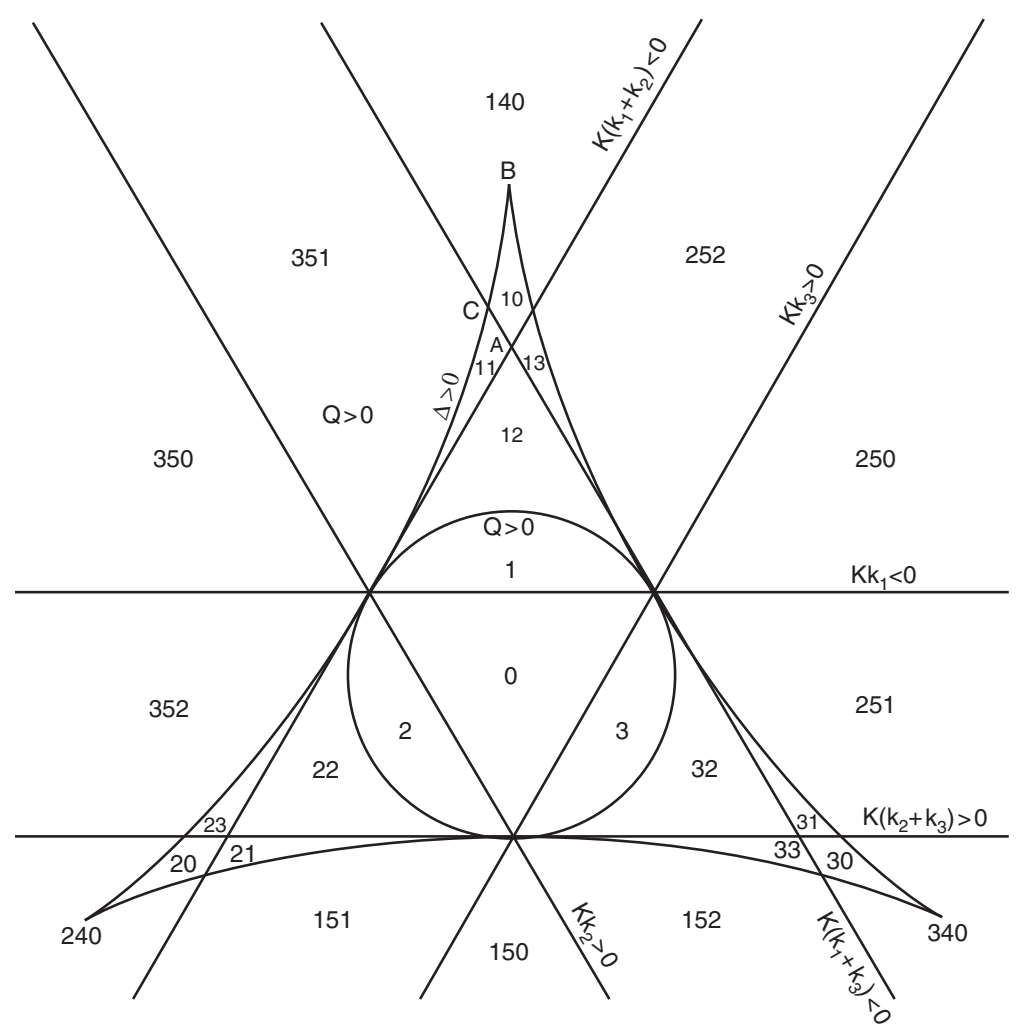

Fig. 1. The strengths space. The numbering of regions reflects the ternary symmetry. 
domain 351

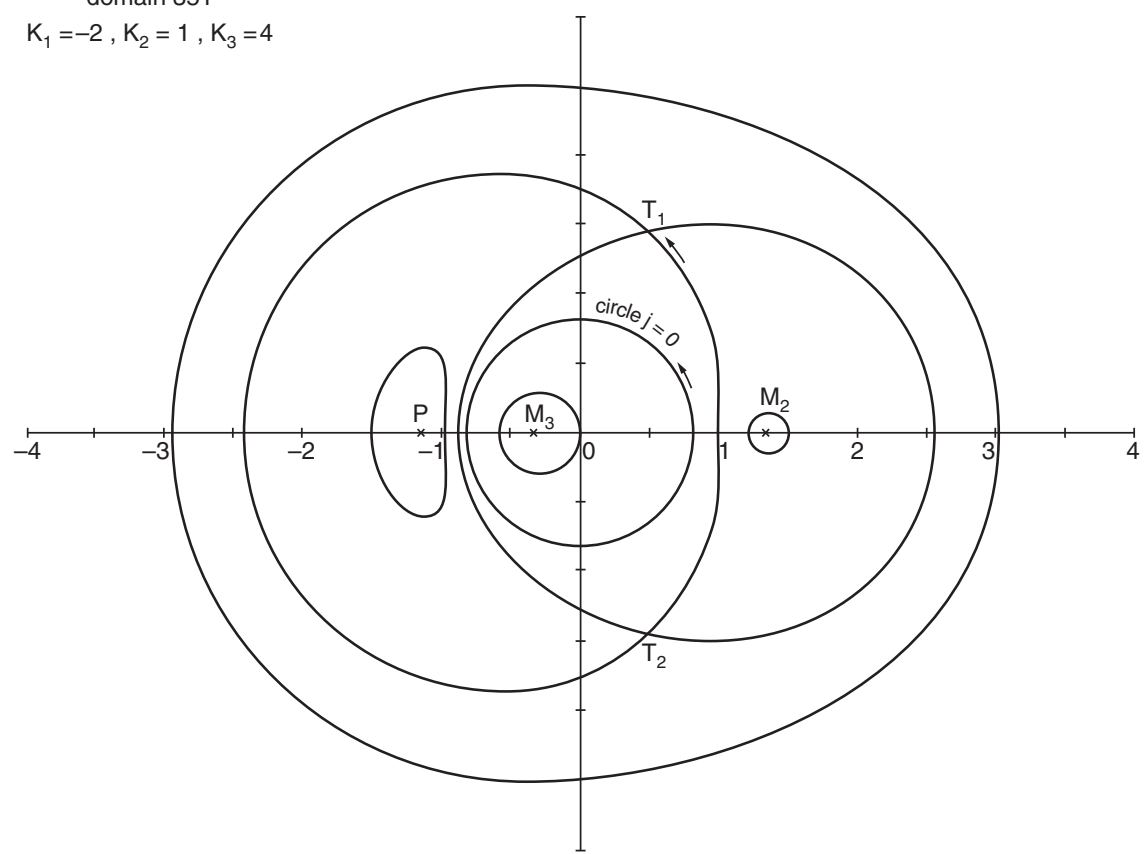

Fig. 2. $\zeta$ plane in the domain $351(Q<0, \Delta>0)$

domain 351

$\kappa_{1}=-2, \kappa_{2}=1, \kappa_{3}=4$.

Since there is in general no stationary point on the orbit, the reduced motion is periodic and the period is expressed by

$$
T=-\frac{\pi J}{s} \oint \frac{\left|\left(\zeta+\kappa_{2}\right)\left(\zeta-\kappa_{3}\right)\right|^{2}}{\left(\kappa_{1}|\zeta|^{2}+\kappa_{2} \kappa_{3} K\right)^{2}} \frac{\mathrm{d}|\zeta|^{2}}{\operatorname{Im}\left(\zeta^{2}+d \zeta\right)}
$$

When $J$ is zero we use (9) instead of (8) to obtain a similar expression, a result which shows that the condition $J=0$ alone represents nothing special as one would believe in the Aref classification (for more details see the third part of this paper). To have a dimensionless result we can take as unit of time $T_{u}=\frac{4 \pi^{2} J}{Q K}$ which is, as we shall see later, the period of the absolute motion when the vortices are in a configuration of relative equilibrium; therefore, in every domain of the multiply-connected $\zeta$ plane, $\frac{T}{T_{u}}$ depends only on $c$. 


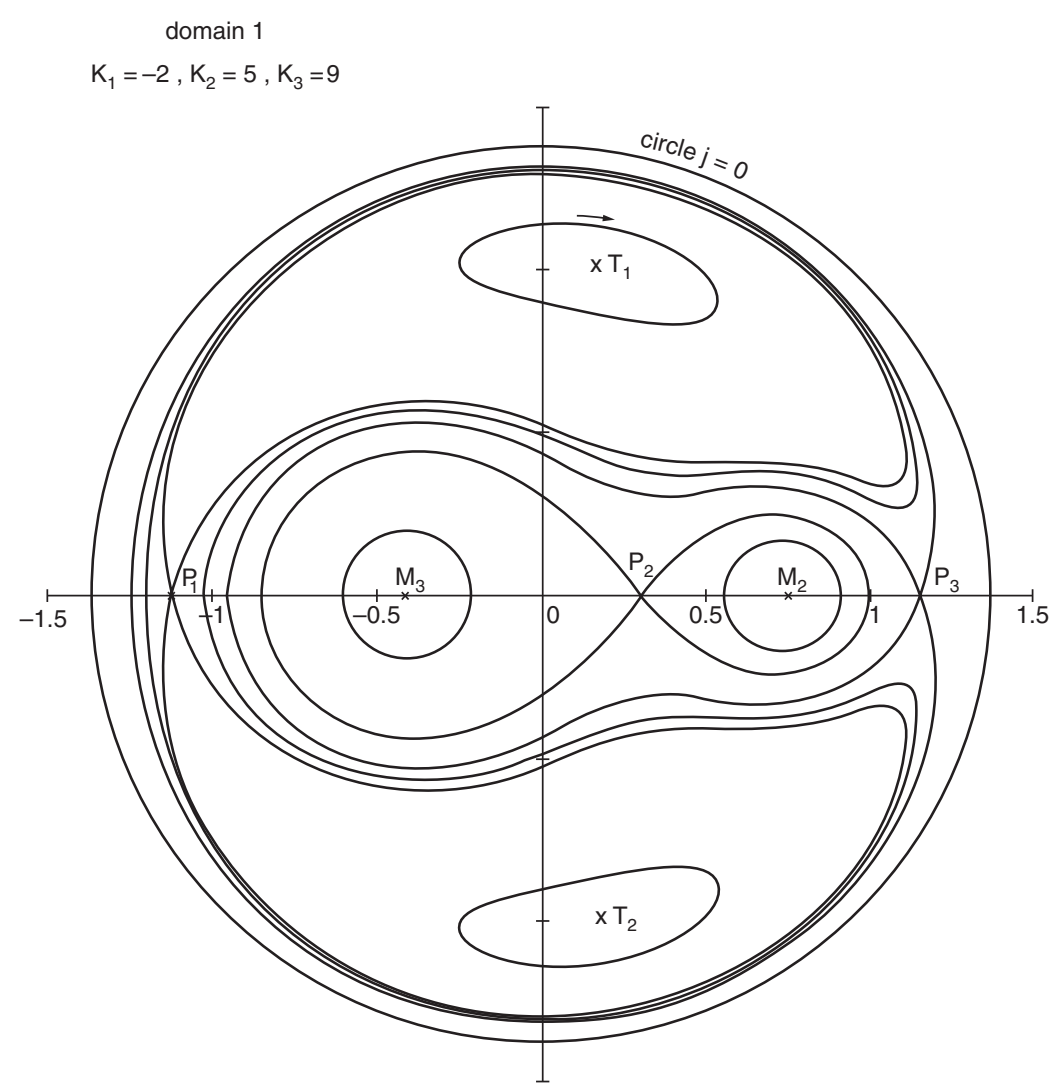

Fig. 3. $\zeta$ plane in the domain $1(Q>0, \Delta<0)$ domain 1 $\kappa_{1}=-2, \kappa_{2}=5, \kappa_{3}=9$.

\section{The absolute motion}

Using the parametric representation of the $z_{j}$ 's we easily obtain the following relations:

$$
\frac{K z_{1}-B}{s \zeta}=\frac{K z_{2}-B}{\kappa_{3} K-\kappa_{1} \zeta}=\frac{K z_{3}-B}{-\kappa_{2} K-\kappa_{1} \zeta} .
$$

Then, using (8) or (9), we conclude that, when the reduced motion is periodic, the modulus of $z_{j}-\frac{B}{K}$ has the period $T$ of the reduced motion. As to the arguments of these affixes, after one period they have all been 


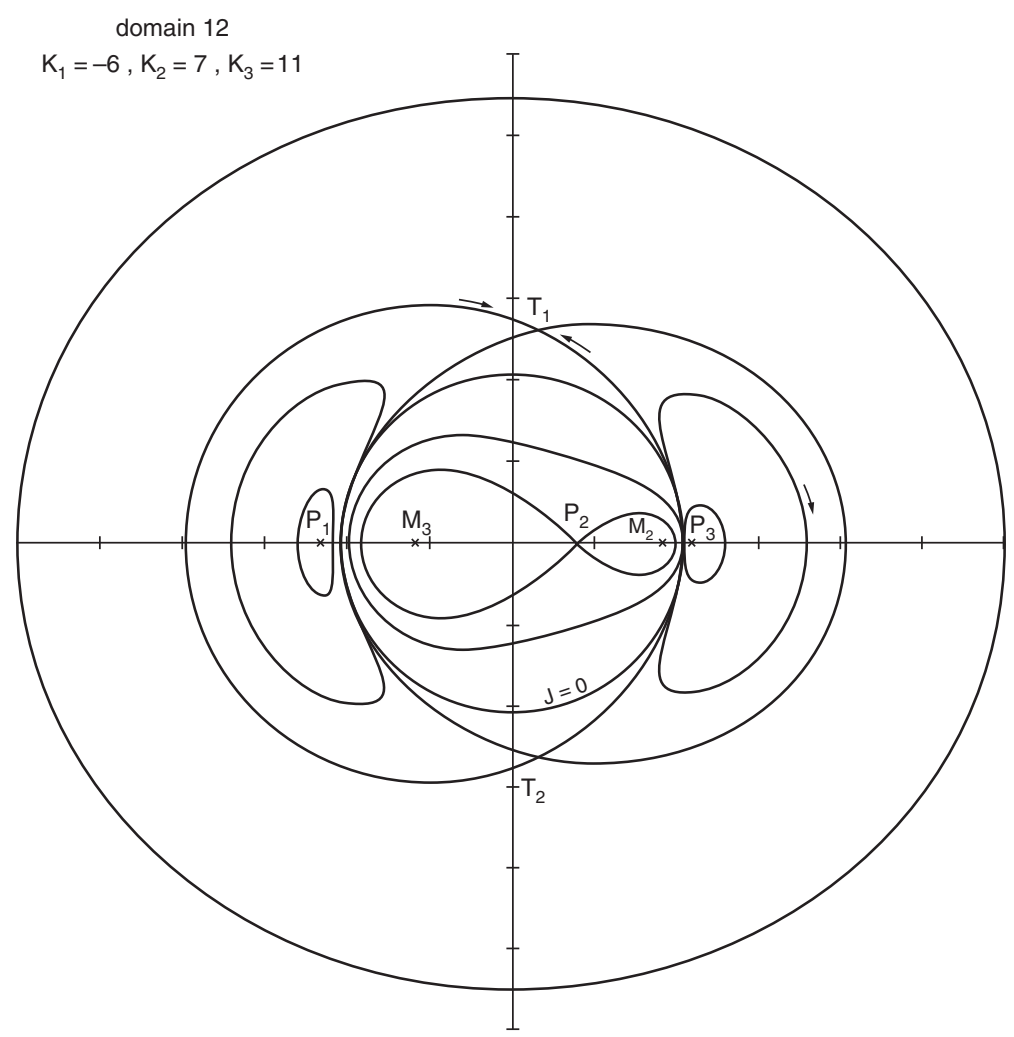

Fig. 4. $\zeta$ plane in the domain $12(Q<0, \Delta<0)$ domain 12 $\kappa_{1}=-6, \kappa_{2}=7, \kappa_{3}=11$.

increased by the same value, modulo $2 \pi$ :

$$
\left\{\begin{aligned}
\Delta \varphi_{1} & =\left[\arg \left(z_{1}-\frac{B}{K}\right)\right]_{o}^{T} \\
& =\oint \frac{-K \operatorname{Re}\left\{\left(\zeta^{2}+d \zeta\right)\left(\bar{\zeta}^{2}+d \bar{\zeta}-\kappa_{2} \kappa_{3}\right)\right\}}{2|\zeta|^{2}\left(\kappa_{1}|\zeta|^{2}+\kappa_{2} \kappa_{3} K\right) \operatorname{Im}\left(\zeta^{2}+d \zeta\right)} \mathrm{d}|\zeta|^{2}=\Delta \varphi \\
\Delta \varphi_{2} & =\Delta \varphi_{1}+\left[\arg \left(\frac{\kappa_{3} K-\kappa_{1} \zeta}{s \zeta}\right)\right]_{o}^{T}=\Delta \varphi \text { modulo } 2 \pi \\
\Delta \varphi_{3} & =\Delta \varphi_{1}+\left[\arg \left(\frac{-\kappa_{2} K-\kappa_{1} \zeta}{s \zeta}\right)\right]_{o}^{T}=\Delta \varphi \text { modulo } 2 \pi
\end{aligned}\right.
$$




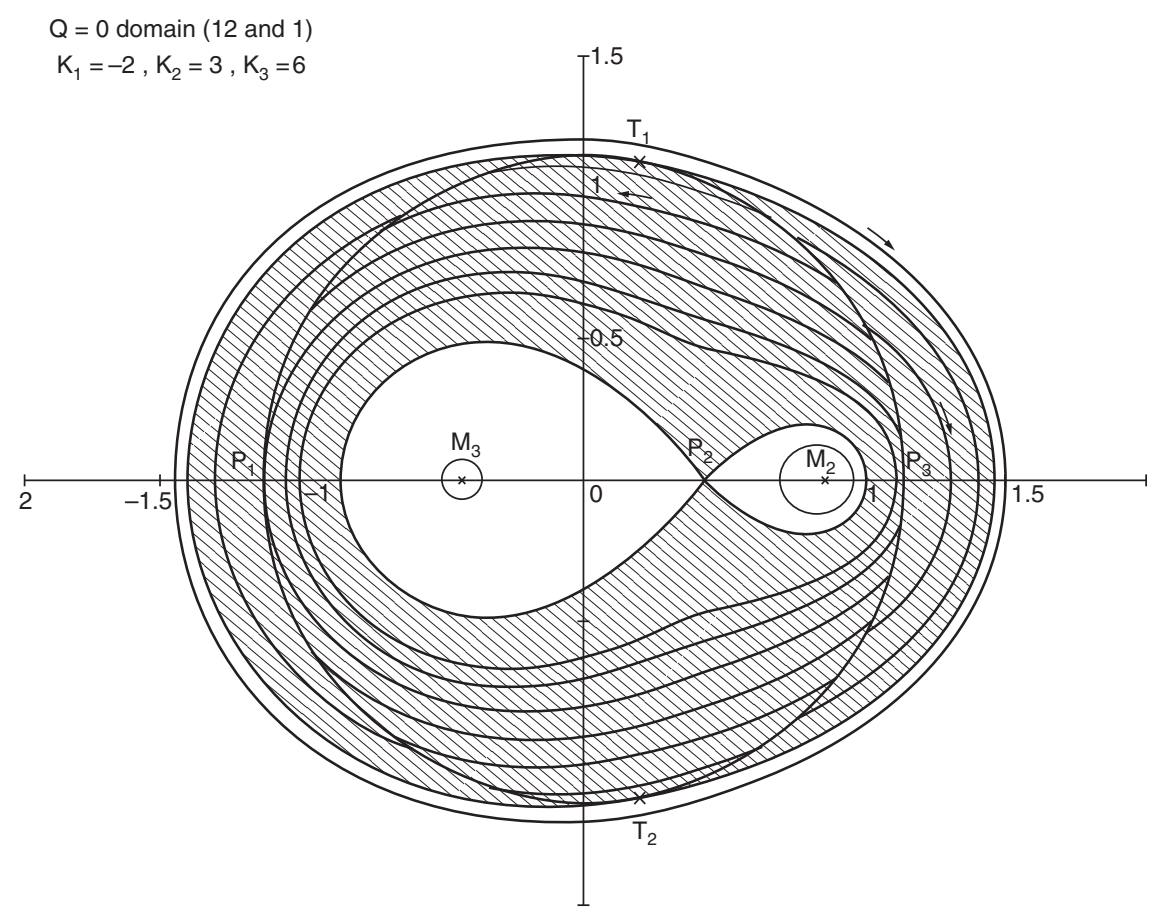

Fig. 2. $\zeta$ plane on the line $Q=0$. The nonperiodic domain is hatched $Q=0$ domain (12 and 1$) \kappa_{1}=-2, \kappa_{2}=3, \kappa_{3}=6$.

For the motion it means that, after a time interval of $T$, the shape and size of the triangle are again the same, i.e. the new positions are deduced from their initial values by a rotation of $\Delta \varphi$ around the center of vorticity. Depending on the domain of initial conditions, the number of turns around the barycentrum may vary from one vortex to another by an integer value. Therefore, when $K$ is nonzero, the absolute motion of any vortex is the product of a uniform rotation about the barycentrum and of a periodic motion, the two periods being the same for the three vortices:

$$
\forall j=1,2,3: z_{j}(t)=\frac{B}{K}+\left(z_{j}(0)-\frac{B}{K}\right) e^{i \Delta \varphi \frac{t}{T}} f_{j}\left(\frac{t}{T}\right),
$$

where $f_{j}$ is periodic with period 1 . In other words, in uniformly rotating axes centered at the barycentrum, the absolute motion is periodic. Figure 8 shows the absolute trajectory of $M_{1}$ both in fixed axes and in rotating axes, for $\vec{\kappa}=(-2,1,4)$ and $\zeta_{o}=-\frac{3 K}{2}$ (the $\zeta$ orbit is the small curve surrounding $P$ in Figure 2). 


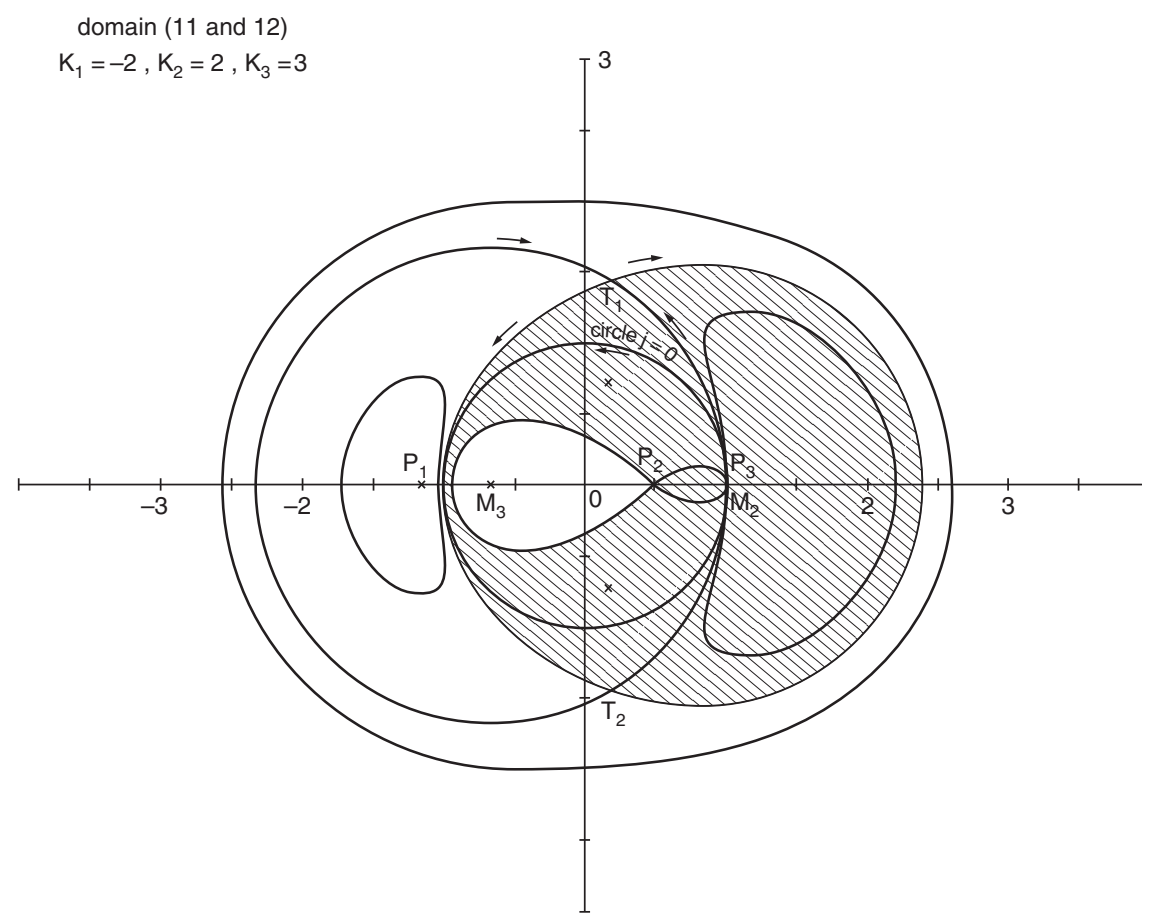

Fig. 3. $\zeta$ plane on the line (11 and 12$)$. The diffusion domain is hatched domain (11 and 12) $\kappa_{1}=-2, \kappa_{2}=2, \kappa_{3}=3$.

Some symmetries may exist in the absolute motion: for instance if the $\zeta$ axis is an axis of symmetry of the $\zeta$ orbit, then at intervals distant of $\frac{T}{2}$ the vortices are colinear; if we choose for origin of time an instant of colinearity, the absolute trajectory of every vortex, when run between 0 and $T$, possesses an axis of symmetry (see Figure 8 ).

Let us now mention an important result concerning this dynamical system: since integral (14) is a continuous function of both the strengths $\frac{\vec{k}}{K}$ and the initial conditions, the angle $\Delta \varphi$ is generically incommensurable with $2 \pi$, i.e. commensurability occurs only for a set of strengths and initial conditions of zero measure. We conclude that generically, due to the incommensurability of $\Delta \varphi$ with $2 \pi$, the trajectory of a given vortex completely fills an annulus centered at the barycentrum (see Landau and Lifchitz Figure 9 Chap. III).

In the case of a vortex plasma $(K=0)$ and when the barycentrum is at infinity $(B \neq 0)$, the variation of $z_{j}$ over one period does not depend on $j$ 


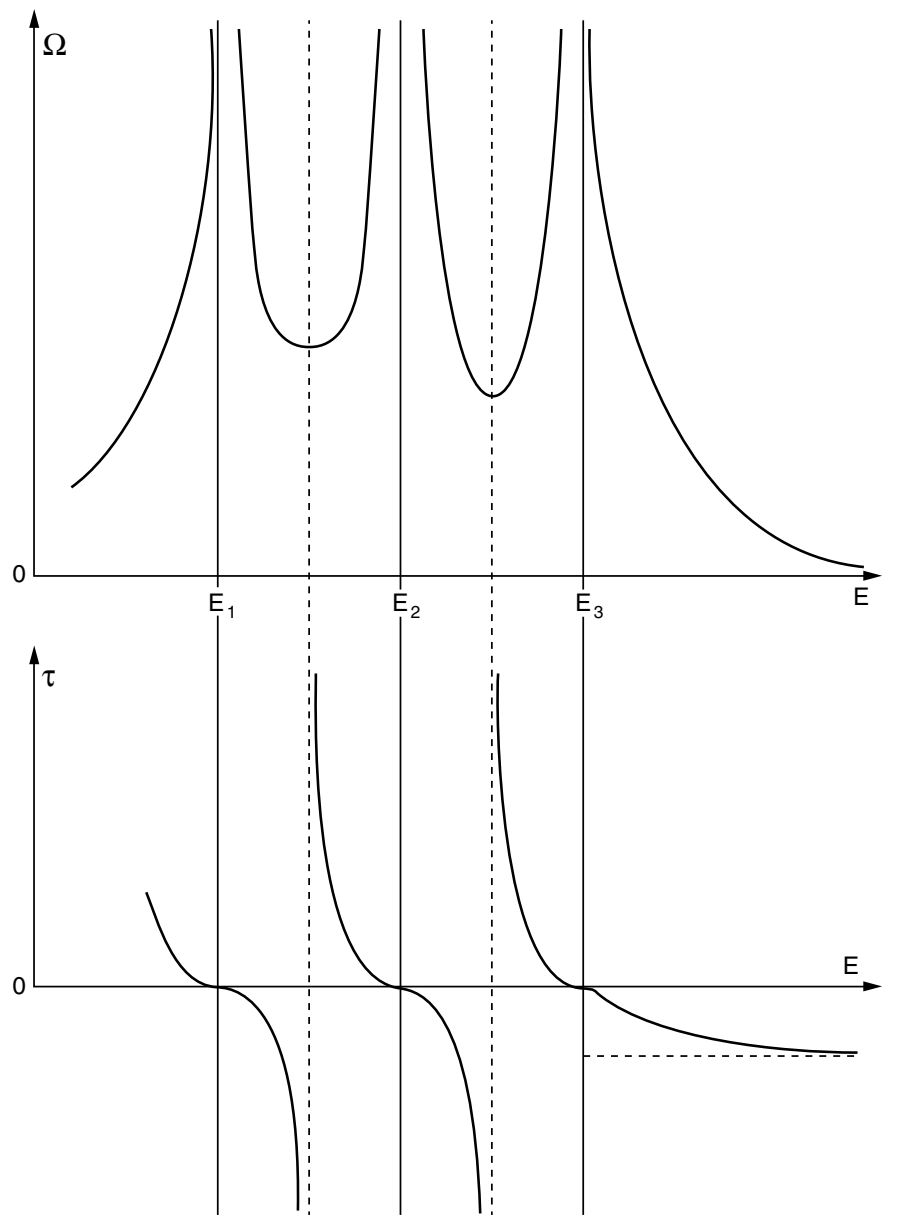

Fig. 7. Volume $\Omega$ and temperature $\tau$ versus energy in the domain $0\left(k_{j} K>0\right)$. Data for these qualitative figures are taken from $\vec{\kappa}=(2,9,13)$.

and is given by

$$
\frac{i}{B}\left[\bar{z}_{j}\right]_{o}^{T}=\oint \frac{\left(\zeta^{2}+d \zeta\right)\left(\bar{\zeta}^{2}+d \bar{\zeta}-\kappa_{2} \kappa_{3}\right)}{2 \kappa_{1}|\zeta|^{4} \operatorname{Im}\left(\zeta^{2}+d \zeta\right)} \mathrm{d}|\zeta|^{2},
$$

which evaluates to a real quantity. The rotation has become a uniform translation in the direction normal to the direction of the impulse. The common mean velocity of the vortices is $\left[z_{1}\right] / T$ and, for instance in the case of an initial equilateral triangle where the absolute motion is a uniform translation, this velocity evaluates to $\frac{i Q}{2 \pi B}$. 


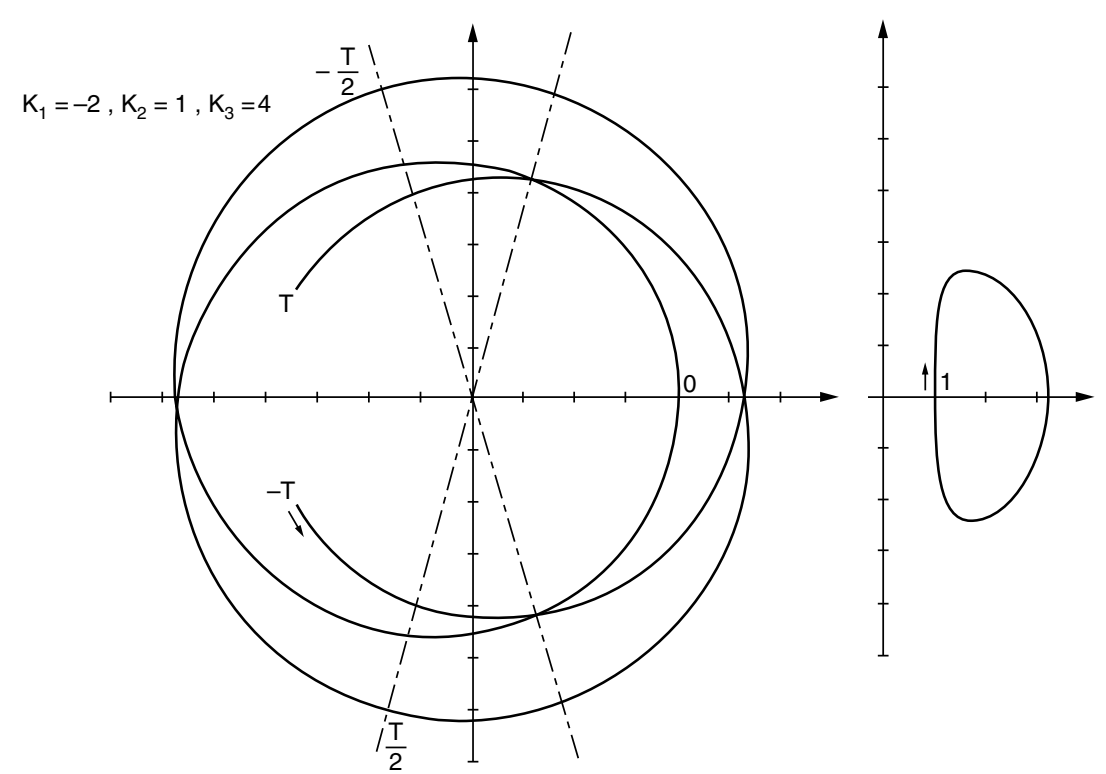

Fig. 8. Absolute motion $z_{1}(t)$ (left) and rotating motion $z_{1}(t) e^{-i \Delta \varphi \frac{t}{T}}$ (right), $-T<$ $t<T$, for arbitrary strengths and initial conditions: $\vec{\kappa}=(-2,1,4), \zeta_{o}=-\frac{3 K}{2}$. Data are: $c=1.726, \frac{T}{T_{u}}=0.262, \Delta \varphi=507.9^{\circ}$.

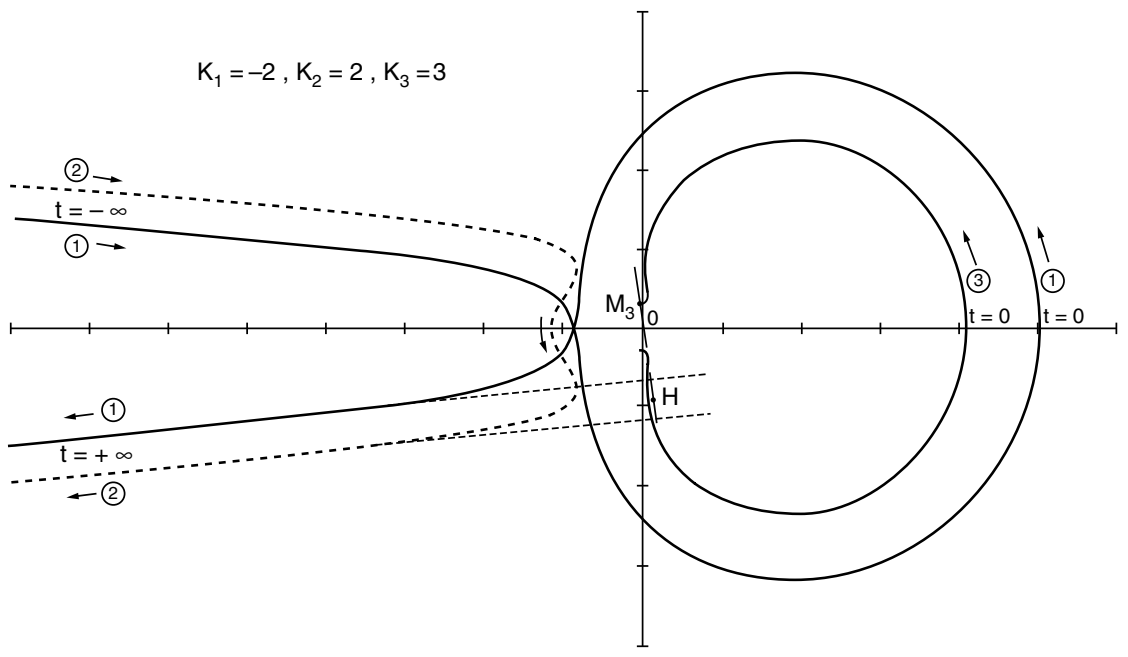

Fig. 9. Absolute motion of the three vortices for an elastic diffusion. $\vec{\kappa}=(-2,2,3)$, $\zeta_{o}=-\frac{20}{21} K(c=1.170)$. 
Let us conclude all that with a geometrical remark: the relations

$$
\kappa_{1}\left|K z_{1}-B\right|^{2}+\kappa_{2} \kappa_{3} K\left|z_{2}-z_{3}\right|^{2}=s J
$$

and

$$
2 \pi \frac{\mathrm{d}\left|z_{2}-z_{3}\right|^{2}}{\mathrm{~d} t}=\frac{2 \kappa_{1} s^{2} \operatorname{Im}\left(\zeta^{2}+d \zeta\right)}{\left|\left(\zeta+\kappa_{2}\right)\left(\zeta-\kappa_{3}\right)\right|^{2}},
$$

show that the extrema of $\left|z_{j}-\frac{B}{K}\right|$ occur simultaneously with those of $\mid z_{\ell}-$ $z_{m} \mid(j, \ell, m$ permutation of $1,2,3)$ and that they are reached when the triangle is either flat or isosceles with $M_{j}$ as a summit.

\section{The absolute motion for two or three equal strengths}

An important practical case is that of the invariance of a $\zeta$ orbit under one of the six permutations of the three elements $\left(\kappa_{j}, z_{j}\right)$. For instance the permutation (132) acts by: $\kappa_{2} \rightarrow \kappa_{3}, \kappa_{3} \rightarrow \kappa_{2}$ and is seen to leave the set of $\zeta$ orbits invariant provided $\kappa_{2}=\kappa_{3}$. Since most of the vortices encountered in nature have equal, or opposite, strengths, we shall consider this particular case in some detail.

Let us therefore assume $\kappa_{2}=\kappa_{3}$ and consider a $\zeta$ orbit having the origin as a center of symmetry (e.g. the circle $J=0$ on the Figure 3 assumed continuously deformed so as to admit the origin as a center of symmetry; note that, on the same figure, the orbit surrounding $T_{1}$ has not the required property). Having chosen such an initial condition $t=0, \zeta=\zeta_{o}$, during the evolution there will happen a time when $\zeta$ evaluates to $-\zeta_{o}$; this time is necessarily equal to the half-period $T / 2$ and, since the states $\left(t=0, \zeta=\zeta_{o}\right)$ and $\left(t=\frac{T}{2}, \zeta=-\zeta_{o}\right)$ are identical from a point of view of initial conditions, we conclude to the identity of the motions for $0<t<\frac{T}{2}$ and for $\frac{T}{2}<t<T$; the triangle at $t=\frac{T}{2}$ is deduced from the triangle at $t=0$ by some fixed rotation around the barycentrum (a change of size and a translation are excluded because of the invariants) and of course by the exchange of 2 and $3(B=0)$ :

$$
\begin{gathered}
\forall t: z_{1}(t)=z_{1}\left(t-\frac{T}{2}\right) e^{i \alpha}, \quad z_{2}(t)=z_{3}\left(t-\frac{T}{2}\right) e^{i \alpha}, \\
z_{3}(t)=z_{2}\left(t-\frac{T}{2}\right) e^{i \alpha} .
\end{gathered}
$$

By iterating, we see that $2 \alpha$ is equal to $\Delta \varphi$ modulo $2 \pi$. Therefore the absolute motions of 2 and 3 are identical, up to a rotation in space and a translation in time. Every absolute trajectory has two independent axes of symmetry and therefore, an infinity: they are defined by the barycentrum 
and the absolute positions of the vortex when the triangle is either isosceles or flat (i.e. when $\zeta$ crosses one of its two symmetry axes).

If $K$ is zero, i.e. $\vec{\kappa}=(-2,1,1)$, the conclusions are similar, with a translation of $L / 2$ instead of a rotation, $L$ being the translation after one period; the initial conditions which have the required symmetry are defined by $0<c<1$.

Let us now study the absolute motion of three identical vortices. The set of orbits is invariant under the six permutations, but a given orbit is only invariant under two or three of them. Two regimes are found:

(1) $\sqrt[3]{2}<c$. The shortest of the three mutual distances is always the same, say $M_{2} M_{3}$, the $\zeta$ orbit is invariant by a permutation of 2 and 3 and the above conclusions apply. The three vortices are colinear at intervals of $T / 2$. Choosing for $t=0$ a colinear configuration, the triangle is isosceles at $t=\frac{T}{4}+n \frac{T}{2}, n \in Z$ and the summit of the triangle is then always the same vortex $M_{1}$.

(2) $1<c<\sqrt[3]{2}$, i.e. a vicinity of the equilateral configurations. The $\zeta$ orbit is invariant under any circular permutation and, using quite similar arguments, we deduce for instance $(B=0)$ :

$$
\begin{gathered}
\forall t: z_{1}(t)=z_{2}\left(t-\frac{T}{3}\right) e^{i \alpha}, \quad z_{2}(t)=z_{3}\left(t-\frac{T}{3}\right) e^{i \alpha}, \\
z_{3}(t)=z_{1}\left(t-\frac{T}{3}\right) e^{i \alpha},
\end{gathered}
$$

with $3 \alpha=\Delta \varphi$ modulo $2 \pi$. This time, the three motions are identical. The vortices are never colinear. Choosing for $t=0$ an isosceles configuration, we see that the triangle regains the same shape at $t=n \frac{T}{3}$, successively with the summits 1,2,3 (or 1,3,2 depending on the initial conditions), and a second isosceles shape at $t=\frac{T}{6}+n \frac{T}{3}$.

The period $T$, that Novikov gave as a hyperelliptic integral, is reducible to an ordinary elliptic integral (see Appendix I).

\section{The relative equilibria and the bifurcation set}

In two fundamental papers linking topology to mechanics, Smale (1970) explains how to split the study of any dynamical system into two simpler problems. He first defines the integral manifolds as the set of points in the phase space with given values of the invariants, or better as the quotient of that set by the symmetry group of the system. Then the first problem is to 
find the topology of the integral manifolds of the phase space and more precisely to find the bifurcation set, i.e. the set of values of the invariants $(E, J)$ for which this topology, and therefore the nature of the motion, changes. The second problem, which has been solved above at least in the general case, is the study of the dynamical systems on the integral manifolds.

Since we do not want to insist on the mathematics, we shall only give the bifurcation set, i.e., for every value of the strengths, we shall determine the values of $E$ and $J$ which cause a qualitative change in the absolute motion; such a research will introduce separating lines in the strengths space.

Our discussion will therefore take place in two different spaces: the space of strengths (parameter space) and the space of invariants, spaces which we are now going to describe in more detail.

Due to the homogeneity of the equations of motion, the strengths space may be represented by its section by a plane $K=$ constant and, in this plane, by a figure invariant under a rotation of $\frac{2 \pi}{3}$ around the point $\kappa_{1}=$ $\kappa_{2}=\kappa_{3}$. Its dimension is therefore 2 and we shall represent a point by its polar coordinates:

$$
\rho \cos \theta=\frac{\sqrt{3}\left(\kappa_{2}-\kappa_{3}\right)}{2 K}, \quad \rho \sin \theta=\frac{-2 \kappa_{1}+\kappa_{2}+\kappa_{3}}{2 K},
$$

$\theta$ describing any interval of amplitude $\frac{2 \pi}{6}$ (see Figure 1).

For given values of the strengths, the space of invariants is a priori bidimensional, since the center of vorticity is not a relevant invariant except when $K$ is zero. Let us compare this space to the space of initial conditions. As we have seen, an initial condition is an orbit in the $\zeta$ plane, since two sets of absolute positions $z_{i}$ whose $\zeta$ values belong to the same $\zeta$ orbit evolve in the same absolute motion, up to a translation in time and a translation, rotation and scale change in space. On a given orbit, $c$ is constant, but, inversely, the equation $c=$ cst represents a finite number (between 0 and 4, see Table 11) of orbits. From this fact, we draw two conclusions: first, the space of invariants $(E, J)$ is in fact of dimension one, two points being identified if they lead to the same value of $c=\frac{J}{Q} e^{4 \pi \frac{E}{Q}}$; second, an initial condition is characterized by, and therefore equivalent to, a value of the invariant $c$ plus an index of region in the $\zeta$ plane (or of sheet in the phase space). We can therefore identify the space of initial conditions to the product of the one-dimensional space of invariants by the finite set of the indices of region. Accordingly, the most precise graphical representation will be the orbits of the $\zeta$ plane, but we shall also use for simplicity a plane $(E, J)$ or 
Table 1. Summary of results.

\begin{tabular}{|c|c|c|c|c|c|c|}
\hline \multirow[b]{2}{*}{ strengths domain } & \multirow[b]{2}{*}{$\operatorname{sign}(\mathrm{Q})$} & \multirow[b]{2}{*}{$\operatorname{sign}(\Delta)$} & \multicolumn{3}{|c|}{ degeneracy } & \multirow[b]{2}{*}{ topology } \\
\hline & & & $c=-\infty$ & $c=0 \quad c=1$ & $c=+\infty$ & \\
\hline 0 & + & - & 0 & 0 & $2,1,2,3$ & $\mathrm{H} \mathrm{M} \mathrm{H} \mathrm{M} \mathrm{H}$ \\
\hline 1 & + & - & 1 & $3,2,1,2$ & 0 & H M H M H \\
\hline 10 & - & - & $0,1,2,1$ & 2 & 2 & M E H E M \\
\hline 11 & - & - & $1,2,1$ & 2 & 2,1 & E M H E M \\
\hline 12 & - & - & 2,1 & 2 & $2,1,0$ & E M H M E \\
\hline 140 & - & + & 0,1 & 2 & 2 & M E M \\
\hline 150 & - & + & 0 & 1,2 & 2 & M E M \\
\hline 151 & - & + & 1 & 2 & 2,1 & M E M \\
\hline$K=0, \kappa_{1}<0<\kappa_{2}<\kappa_{3}$ & - & + & 0 & 2 & 2 & M E M \\
\hline$\Delta=0(11$ and 351$)$ & - & 0 & 1,1 & 2 & 2,1 & E M P M \\
\hline$\Delta=0(10$ and 140$)$ & - & 0 & $0,1,1$ & 2 & 2 & M E P M \\
\hline$\prod\left(\kappa_{j}+\kappa_{l}\right)=0(11$ and 12$)$ & - & + & 2,1 & 2 & 2,1 & E M H X \\
\hline id. (10 and 11$)$ & - & + & $1,2,1$ & 2 & 2 & X H E M \\
\hline id. (140 and 351) & - & - & 1 & 2 & 2 & X M \\
\hline$Q=0(1$ and 12$)$ & 0 & - & 0 & $2,1,2,4$ & 1 & M H M \\
\hline point B $(-5,4,4)$ & - & 0 & 0,1 & 2 & 2 & M Q M \\
\hline point $\mathrm{A}(-1,1,1)$ & - & - & 2,2 & 3 & 2 & X H X \\
\hline point $\mathrm{C}(-2, \sqrt{3}, 2)$ & - & 0 & 1,1 & 2 & 2 & X P M \\
\hline
\end{tabular}

The strengths domains are defined in Figure1 Under the heading "degeneracy" are listed by interval of $c$ the number of different states, i.e. of $\zeta$ orbits, having the same value of $c$; for instance, in the domain 11, the limiting values of $c$ are $-\infty, 0,+\infty$ (at the points $M_{j}$ ), 1 (at the triangles), $c_{1}, c_{2}, c_{3}$ (at the colinear relative equilibria), they define 6 intervals, hence a sequence of 6 degeneracy numbers; when $Q$ is zero, $e^{4 \pi E / K^{2}}$ is used in place of $c$ for the classification.

The column "topology" lists the sequence of the nature of the real remarkable $\zeta$ points along the real axis: $M$ stands for a point $M_{j}$ (we omit $M_{1}$ at infinity), $E, H, P$ for elliptic, hyperbolic, parabolic, $X$ for an $M$ coinciding with $E$ or $H$ and $Q$ for the only higher-order point we found. The information on the nature of the triangles is contained in the column sign $(Q)$. All this is sufficient to draw every $\zeta$ plane. 
an axis $c$ with some handwritten information on it to take into account the integer index.

Let us now proceed to the determination of the bifurcation set. We exclude the set of collisions, represented in the $\zeta$ plane by three points of affixes $\infty, \kappa_{3},-\kappa_{2}$ where $c$ evaluates to $+0,-0,+\infty$ or $-\infty$ depending on the signs of $J, Q$ and the strengths; these limiting values of $c$ will therefore belong to the bifurcation set.

The nature of the motion (at least its topological nature since it is always generically biperiodic) changes when there is a stationary point on the $\zeta$ orbit; such points, where the velocity of $\zeta$ vanishes, are given by the solutions of the complex equation

$$
\bar{\zeta}\left(\zeta^{2}+d \zeta-Q\right)-s K(\zeta+d)=0
$$

equivalent to

$$
\frac{K \bar{z}_{1}-\bar{B}}{z_{2}-z_{3}}+\frac{K \bar{z}_{2}-\bar{B}}{z_{3}-z_{1}}+\frac{K \bar{z}_{3}-\bar{B}}{z_{1}-z_{2}}=0 .
$$

These points are also the critical points of the function $(\xi, \eta) \rightarrow c$ and they give all the relative equilibrium configurations of the three vortices, i.e. the states for which the system moves generally like a rigid body. The solutions of (17) are:

(a) one or three colinear configurations, defined by the real zeros of $P(\zeta) \equiv$ $\zeta^{3}+d \zeta^{2}-(s K+Q) \zeta-s K d$ (points $P_{1}, P_{2}, P_{3}$ of Figures 2 to 3 ),

(b) two equilateral triangles, defined by the zeros of $\zeta^{2}+d \zeta+s K-Q$, i.e. $\zeta=\frac{-d \pm i s \sqrt{3}}{2}$ (points $T_{1}$ and $T_{2}$ of Figures 2 to 3 ); these stationary triangles, already known to Kelvin for identical vortices, therefore exist for vortices of any strengths.

(c) When $Q=0$, the isolated zero $\zeta=-d$ of $P$ and every point of the circle $|\zeta|^{2}=s K$ on which lie the summits of the equilateral triangles and the two other real zeros of $P$. On this circle $J$ is equal to zero.

The corresponding absolute motions will be described later. It is interesting to remark that, except for some values of the strengths like for instance $Q=0$, the relative equilibria of the three vortex system are qualitatively the same than those of the three body problem of celestial mechanics, where there are two equilateral triangles and three colinear configurations; in fact, a simple geometric reasoning shows the same qualitative composition of the set of relative equilibria for every planar three body motion ruled by a two body central interaction. 
The study of the stability of the relative equilibria is given in Appendix II and only three different behaviours are found (in celestial mechanics, only two cases arise: the three colinear configurations are always unstable, the two triangles are stable for $\left(m_{1}+m_{2}+m_{3}\right)^{2}-27\left(m_{1} m_{2}+\right.$ $\left.m_{2} m_{3}+m_{3} m_{1}\right)>0$, unstable otherwise), depending on the signs of two polynoms of the strengths of even degree:

$\Delta>0, Q<0$ : unstable triangles, one stable aligned configuration,

$\Delta<0, Q<0$ : unstable triangles, two stable aligned configurations, one unstable,

$\Delta<0, Q>0$ : stable triangles, three unstable aligned configurations,

with

$$
\Delta=-32 \sum_{j \neq l} \kappa_{j}^{3} \kappa_{l}-61 \sum_{j<l} \kappa_{j}^{2} \kappa_{l}^{2}-118 \kappa_{1} \kappa_{2} \kappa_{3} K .
$$

The important role played by $Q$ can easily be understood if we notice that the angular momentum of the system $\sum \kappa_{j}\left(x_{j} \frac{\mathrm{d} y_{j}}{\mathrm{~d} t}-y_{j} \frac{\mathrm{d} x_{j}}{\mathrm{~d} t}\right)$ is precisely $\frac{Q}{2 \pi}$. Like for the planar three body problem (see Smale II), we are going to see that its sign is a basic element of classification of the topology of the phase space and that very special behaviours of the motion occur when it vanishes.

¿From (7) we see that there is no other $\zeta$ orbit where the nature of the motion changes. For given values of the strengths, the bifurcation set is therefore the union, in the $\zeta$ plane, of the three points $\infty, \kappa_{3},-\kappa_{2}$ and the three or five orbits going through the stationary points (see Figures 2 to 4. where some other ordinary orbits have been added). Note that the circle $|\zeta|^{2}=-\frac{\kappa_{2} \kappa_{3} K}{\kappa_{1}}$ on which $J$ is zero does not belong to the bifurcation set, except when $Q$ is zero as we shall see. However, when we represent the bifurcation set in the $(E, J)$ plane, the line $J=0$ seems to belong to it for it is for some values of the strengths the limiting curve $c \rightarrow 0$ associated with two vortices at the same location; Figure 10 shows the same bifurcation set as Figure 3, but represented in the plane $(E, J)$.

Special behaviour for the motion will be obtained when two or more elements of the bifurcation set come into coincidence. This will give us separating lines in the strengths plane. The only conditions of coincidence between the solutions of (17) and the set (d) of collisions $\left(\zeta=\infty, \kappa_{3},-\kappa_{2}\right)$ are:

(i) coincidence of (a) and (d): $\prod_{j \neq l}\left(\kappa_{j}+\kappa_{l}\right)=0$,

(ii) coincidence of two zeros of $P: \Delta=0$. 


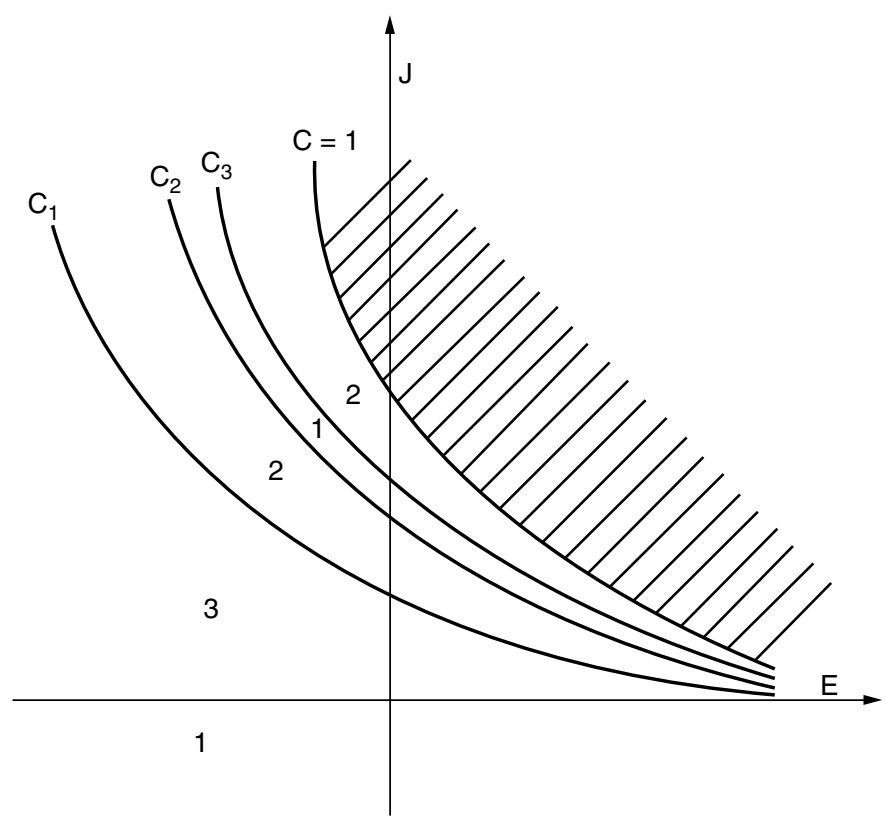

Fig. 10. Bifurcation set in the domain $1(Q>0, \Delta<0)$, not to scale. The numbers are the number of periodic solutions in each region.

We must add to the above mentioned lines the three lines given by $\kappa_{1} \kappa_{2} \kappa_{3}=0$ which are forbidden. $\prod\left(\kappa_{j}+\kappa_{l}\right)$ is represented by three lines, $Q=0$ by the circle $\rho=1$ and $\Delta=0$ by a quartic of equation:

$$
\rho^{4}+8 \rho^{3} \sin 3 \theta+18 \rho^{2}-27=0
$$

the form of which reflects the ternary symmetry.

The intersections of these lines define three distinct remarkable points that we shall study explicitly:

$$
\begin{aligned}
& \text { point A: } \left.\rho=2, \quad 3 \theta=\frac{3 \pi}{2} \text { (strengths }-1,1,1\right), \\
& \text { point B: } \left.\rho=3, \quad 3 \theta=\frac{3 \pi}{2} \text { (strengths }-5,4,4\right), \\
& \text { point C: } \left.\rho=\sqrt{5}, \quad \sin 3 \theta=\frac{-11 \sqrt{5}}{25} \text { (strengths }-2, \sqrt{3}, 2\right) .
\end{aligned}
$$

The points A, B and some numerical values of the strengths correspond to cases of integrability for the period (see Appendix I). 
These lines define different domains in the strengths space, which has been represented on Figure 1. When we stay in one of these domains, the topology of the bifurcation set does not change, and Table 1 summarizes the results concerning the bifurcation set for all domains of the strengths space.

The classification for the bifurcation set is mainly based on the signs of $Q$ and $\Delta$, i.e. on the number and stability of the relative equilibria; among the three quantities, i.e. arithmetic, geometric and harmonic means of the strengths, whose signs were proposed by Aref as a basis for a classification, only the third one $\frac{Q}{3 \kappa_{1} \kappa_{2} \kappa_{3}}$ is relevant, although the factor $\kappa_{1} \kappa_{2} \kappa_{3}$ prevents it from being invariant under a strengths reversal, an operation equivalent to a time reversal which must leave invariant any candidate to a classification; moreover, for any $N$, the formula $\sum^{\prime} \kappa_{j} \kappa_{l}=0$, not $\sum \frac{1}{\kappa_{j}}=0$, expresses the associated physical property of invariance of the energy under a change of length.

In the next part, we describe the behaviour of the three vortex system when it is nongeneric, i.e. when either the strengths are on the boundaries of the domains represented on Figure 1 or when the initial conditions are those of a relative equilibrium. We shall proceed by studying first the relative equilibria associated with nonsingular strengths, then the strengths on the separating lines of the strengths space and finally the three particular points A, B, C of the strengths space.

\section{Absolute motions for nongeneric strengths or initial conditions}

\section{Absolute motions for the ordinary relative equilibria}

Ordinary means that we exclude $\zeta$ points which are the coincidence of two elements of the bifurcation set: this is equivalent to $J$ nonzero and $\zeta$ not a multiple zero of $P$.

For an initial condition in the vicinity of such a $\zeta$ point, every point obeys the general motion, except those lying on the two curves intersecting at an unstable $\zeta$ point, in which case the motion is asymptotic to the motion at the stationary point, motion which we are going to determine:

(a) $K \neq 0$. From the time variation law

$$
2 \pi \frac{\mathrm{d}\left|K z_{1}-B\right|^{2}}{\mathrm{~d} t}=\frac{-2 s^{2} \operatorname{Im}\left(\zeta^{2}+d \zeta\right)}{\left|\left(\zeta+\kappa_{2}\right)\left(\zeta-\kappa_{3}\right)\right|^{2}},
$$


it follows that the distance from each point to the barycentrum remains constant. The absolute motion is therefore a solid rotation around the barycentrum; moreover, using

$$
\sum_{j=1}^{N} \kappa_{j} z_{j} \frac{\mathrm{d} \bar{z}_{j}}{\mathrm{~d} t}=\frac{Q}{2 \pi i}
$$

whose imaginary part yields

$$
\sum_{j=1}^{N} \kappa_{j}\left|z_{j}\right|^{2} \frac{\mathrm{d} \arg z_{j}}{\mathrm{~d} t}=\frac{Q}{2 \pi},
$$

we see that the common angular velocity of every vortex is independent of time and remains equal to $\frac{Q}{2 \pi I_{o}}$, where $I_{O}$ is the inertia momentum relative to the barycentrum

$$
I_{o}=\sum \kappa_{j}\left|z_{j}-\frac{B}{K}\right|^{2}=\frac{J}{K},
$$

and $\frac{Q}{2 \pi}$ is the angular momentum of the system.

The period of the uniform rotation is therefore

$$
T_{u}=\frac{4 \pi^{2} J}{Q K},
$$

a formula valid for any number of vortices when there exists a solid rotation.

A particular case is $Q=0$, for which the only isolated relative equilibrium is $\zeta=-d$, i.e. $\kappa_{2} \kappa_{3} z_{1}+\kappa_{3} \kappa_{1} z_{2}+\kappa_{1} \kappa_{2} z_{3}=0$; all the vortices remain at rest. This situation is of course unstable (free vortices cannot have a stable rest position since the complex velocity is a nonconstant meromorphic function of the affixes), and the small motions have for pulsation $\omega= \pm \frac{9 i \kappa_{1} \kappa_{2} \kappa_{3}}{2 \pi J}$

(b) $K=0$. The three stationary points are ordinary.

$\zeta=0$, stable: the impulse $B$ is zero and the affixes verify:

$$
\frac{z_{2}-z_{3}}{\kappa_{1}}=\frac{z_{3}-z_{1}}{\kappa_{2}}=\frac{z_{1}-z_{2}}{\kappa_{3}} .
$$

The inertia momentum is nonzero and is the same at every point, and the absolute motion is a uniform solid rotation of period

$$
T=\frac{4 \pi^{2} I}{Q}
$$

equal to that of the small motions. 
At the two unstable equilateral triangles, $B$ is nonzero and the absolute motion is a uniform translation of velocity

$$
\forall j: \frac{\mathrm{d} z_{j}}{\mathrm{~d} t}=\frac{i Q}{2 \pi \bar{B}},
$$

orthogonal to the impulse.

\section{Absolute motions on the boundaries of the strengths domains}

These lines are: $Q\left(\kappa_{2}+\kappa_{3}\right)\left(\kappa_{3}+\kappa_{1}\right)\left(\kappa_{1}+\kappa_{2}\right) \Delta=0$.

\section{$Q=0$. Triple collision in a finite time, expanding motion}

In addition to the already studied isolated stationary point, there exists a circle of stationary $\zeta$ points (see Figure 2), on which $J$ is zero, On this circle lie the two summits of the equilateral triangles and the two other zeros of $P$. These four points are the points of contact of the circle $J=0$ with the set of $\zeta$ trajectories whose equation is now:

$$
\left|\frac{\zeta-\kappa_{3}}{s}\right|^{-2 \frac{\kappa_{1} \kappa_{2}}{K_{2}}}\left|\frac{\zeta+\kappa_{2}}{s}\right|^{-2 \frac{\kappa_{1} \kappa_{3}}{K_{2}}}=e^{\frac{4 \pi E}{K^{2}}} .
$$

We shall first study the absolute motion when the $\zeta$ point lies on the circle $J=0$, then examine its neighborhood and finally deduce the bifurcation set.

Let us assume $\zeta$ on the circle $J=0$. This circle is no longer a trajectory and the $\zeta$ point stays at rest. The two conditions $Q=0, J=0$ express that both the energy and the inertia momentum relative to the barycentrum are invariant under a change of length, and therefore nothing prevents the vortices from going to infinity or to zero; we see below that both cases are possible. The absolute motion is ruled by Equation (6) which implies that $\frac{d \rho_{1}^{2}}{d t}$ and $\rho_{1}^{2} \frac{d \varphi_{1}}{d t}$ are constant in time $\left(\rho_{1}, \varphi_{1}\right.$ are polar coordinates of $M_{1}, B$ is chosen zero). Since the shape of the triangle is conserved, Equation (6) integrates as in

$$
\begin{aligned}
\forall j & =1,2,3: z_{j}=z_{j, o}\left(1-\frac{t}{t_{c}}\right)^{1 / 2-i \omega t_{c}}, \\
\text { i.e. } \sqrt{1-\frac{t}{t_{c}}} & =\frac{\rho_{j}}{\rho_{j, o}}=\exp \left\{\left(\varphi_{j}-\varphi_{j, o}\right) /\left(-2 \omega t_{c}\right)\right\},
\end{aligned}
$$


where the characteristic time $t_{c}$ and the initial angular velocity $\omega$ are defined by

$$
-2 \omega+\frac{i}{t_{c}}=\frac{-s^{2}}{\pi K\left|z_{1,0}\right|^{2}} \frac{\zeta^{2}+d \zeta}{\zeta^{2}+d \zeta-\kappa_{2} \kappa_{3}}=\frac{1}{3 \pi} \sum_{j, \ell}^{\prime} \ldots \frac{\kappa_{\ell}}{\left(\bar{z}_{j}\left(z_{\ell}-z_{j}\right)\right)_{o}} .
$$

$\omega$ never vanishes and has the sign of $K \cdot \frac{1}{t_{c}}$ vanishes and changes sign when $\zeta$ is one of the four points already mentioned where the circle is tangent to the set of $\zeta$ curves. We conclude that, for a $\zeta$ point of the circle $J=0$ distinct of these four points, every vortex runs a logarithmic spiral whose pole is the barycentrum, the shape of the triangle remains constant and, depending on the sign of $t_{c}$, the triangle either expands to infinity in an infinite time or collapses on the barycentrum in a finite time $t_{c}$. At the time of this triple collision all the denominators of the equations of motion (1) simultaneously vanish like $\left(t_{c}-t\right)^{1 / 2}$. After the collision, the system is made of a single motionless vortex of strength $K$ located at what was the center of vorticity.

When $\frac{1}{t_{c}}$ vanishes, the spiral motion degenerates into a uniform circular motion whose period $T=\frac{2 \pi}{\omega}$ can also be written as

$$
T=\frac{4 \pi^{2}}{6 K}\left(\left|z_{2}-z_{3}\right|^{2}+\left|z_{3}-z_{1}\right|^{2}+\left|z_{1}-z_{2}\right|^{2}\right),
$$

for the two aligned configurations and twice the same expression for the equilateral triangles.

Let us now examine the motion elsewhere in the $\zeta$ plane $(J \neq 0)$. Figure 2 shows that two generic situations exist depending on whether the $\zeta$ orbit intersects or not the circle $J=0$ of singular points; the limiting $\zeta$ orbits, which belong to the bifurcation set, are $E=0\left(\right.$ tangent at $\left.T_{1}, T_{2}\right)$ and $E=E\left(P_{3}\right)$. For an energy $E$ outside the interval $\left[E\left(P_{3}\right), 0\right]$, the motion is the usual biperiodic one.

Inside the energy interval $] E\left(P_{3}\right), 0[$, striped on Figure 2 every $\zeta$ trajectory stops on the circle (note that it cannot cross it) and, since $J$ is the product of $\left|z_{2}-z_{3}\right|^{2}$ by a function of $\zeta$ vanishing on the circle, every $\zeta$ point having a nonzero $J$ and the energy of a curve intersecting the circle yields an expanding motion in which the trajectory of every vortex is asymptotic to a logarithmic spiral going to infinity. No motion exists which is asymptotic to the triple collision in a finite time: therefore the points of the half circonference (from $P_{1}$ to $T_{1}$ and from $P_{3}$ to $T_{2}$ ) where such a collision exists are repulsive points, while the other half is made of attractive points.

Finally, the bifurcation set, which we have also represented in the $(E, J)$ plane on Figure 11 is the union of the following lines of the $\zeta$ plane: the set of collisions $\left(\zeta=\infty,-\kappa_{2}, \kappa_{3}\right)$, the circle $J=0$ (not an orbit), the orbit 


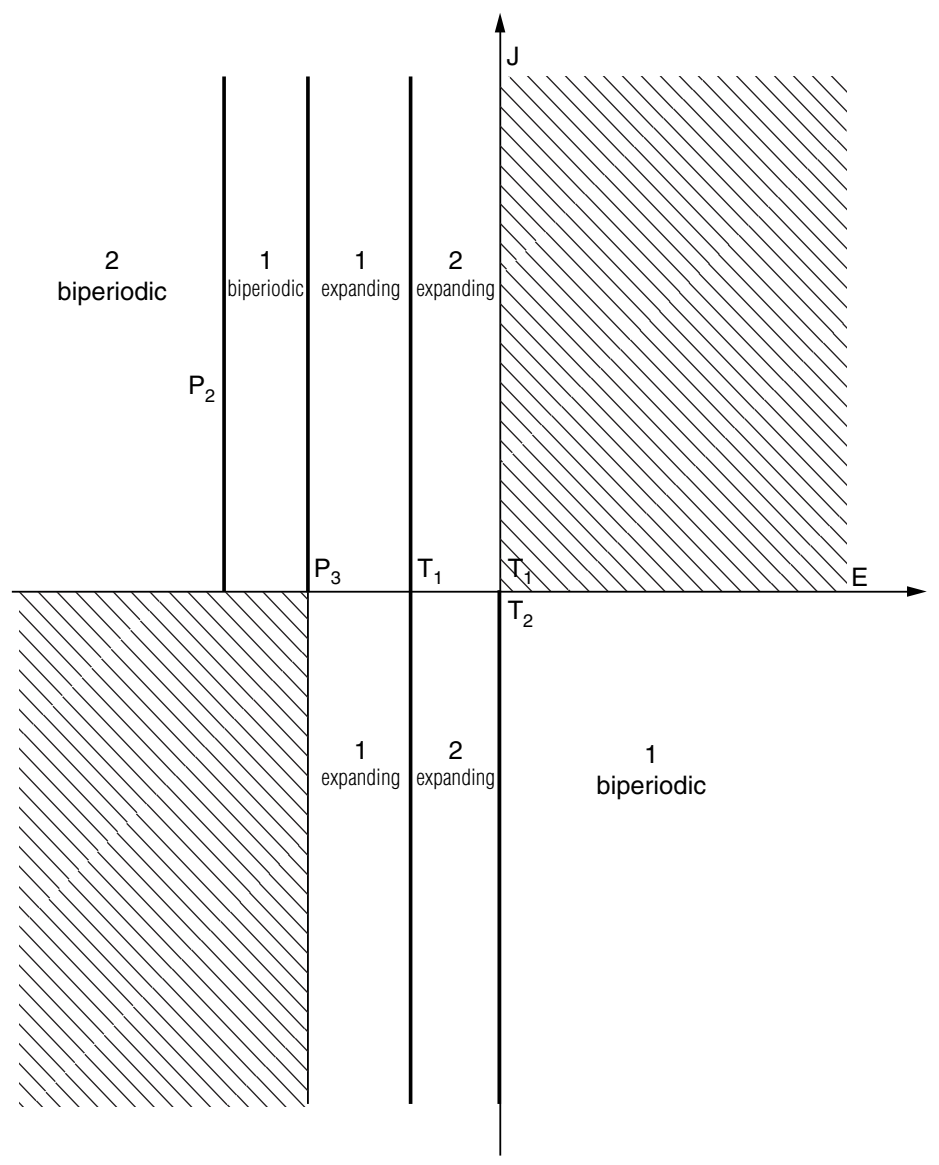

Fig. 11. Bifurcation set for $Q=0$, not to scale.

going through $P_{2}$, the orbit $E=0$ tangent to the circle at $T_{1}, T_{2}$, the orbit $E=E\left(P_{3}\right)$ tangent to the circle at $P_{3}$ and that portion of the orbit $E=E\left(P_{1}\right)$ which is interior to the circle $J=0$.

\section{$\Delta=0$}

Two of the three colinear relative equilibria coincide except at the three points of retrogression $\rho=3$ of the quartic $\Delta=0$ (strengths $-5,4,4$ ) where the three of them coincide and at the six points $\rho=\sqrt{5}$ (strengths $-2, \sqrt{3}, 2$ ) where two of the strengths are opposite (see next case). 
For the absolute motion, there is nothing qualitatively changed: in the vicinity of the double point $\zeta_{o}$, the orbits are equivalent to the cubics of equation

$$
c-c_{o}+A_{2} \eta^{2}+A_{3}\left(\xi-\xi_{o}\right)^{3}=0,
$$

and the period, which behaves like $J \int \frac{\mathrm{d} \xi}{\eta}$, diverges like $\left|c-c_{o}\right|^{-1 / 16}$; the absolute motion is still biperiodic, although the triangle spends quite a long time in nearly aligned states. On the orbit $c=c_{o}$ passing through the unstable $\zeta_{o}$ point, the absolute motion is asymptotic to the usual circular motion, the time law being different: $\xi-\xi_{o} \sim t^{-2}, \eta \sim t^{-3}$.

\section{$\Pi\left(\kappa_{j}+\kappa_{\ell}\right)=0$. Elastic diffusion}

At least two vortices have opposite strengths. To fix the ideas, let us assume $\kappa_{1}+\kappa_{2}=0$. The case $\vec{\kappa}=(-1,1,1)$ will be described in next section. The point $\zeta=\kappa_{3}$, which lies on the circle $J=0$, is singular in the sense that for every $c$ value there exists a $\zeta$ orbit passing through $\kappa_{3}$, which forbids a reduced periodic motion.

Therefore two generic situations exist, as shown on Figure 3 outside of the striped domain which contains $J=0$, the absolute motion is biperiodic. Inside this domain, for every $c$ value there is one and only one $\zeta$ orbit and the $\zeta$ point is attracted by $\kappa_{3}$ in an infinite time: it tends to $\kappa_{3}$ normally to the real axis following a law

$$
i\left(\zeta-\kappa_{3}\right) t \rightarrow \frac{2 \pi s}{\kappa_{1}} e^{-4 \pi E / Q} .
$$

In the absolute space, the point $M_{3}$ stops and the two others go to infinity together with a motion asymptotic to a uniform translation in the direction normal to the line joining the final position of $M_{3}$ to the barycentrum, exactly as if they were alone and obeyed the motion of two vortices of opposite strengths: at the limit, the translation velocity is $\frac{\sqrt{-Q}}{2 \pi} e^{2 \pi E / Q}$ and the mutual distance is $e^{-2 \pi E / Q}$.

This creation of a doublet can also be interpreted in terms of the elastic diffusion of the doublet by the third vortex: if we take for initial condition $\zeta$ near to $K_{3}$ with $\eta / K$ positive, the vortices 1 and 2 are initially a doublet moving towards the third vortex; then the three mutual distances become the same order of magnitude, i.e. there is interaction; finally, the doublet emerges and goes to infinity away from the third vortex with unchanged mutual distance and velocity, but in a different direction. This is exactly a process of elastic diffusion. Figure 9 shows typical absolute motions. We 
define the scattering angle $\Delta(\phi)$ by the total variation along the trajectory of $\arg \left(z_{1}\right)$ ( $B$ is chosen zero) and a dimensionless impact parameter by $\frac{\overline{O H}}{\overline{O M_{3}}}$ (Figure 9) where $H$ is the projection of the middle of $M_{1} M_{2}$ on the line defined by the barycentrum and the final position of $M_{3}$ :

$$
\begin{aligned}
\frac{\overline{O H}}{\overline{O M_{3}}} & =\frac{\operatorname{Re}\left(\bar{z}_{3} \frac{z_{1}+z_{2}}{2}\right)}{\bar{z}_{3} z_{3}} \\
& =\frac{\operatorname{Re}\left(\left(\vec{\zeta}_{3}-\kappa_{3}\right)\left(\kappa_{3}^{2}-\kappa_{1} \zeta+s \zeta\right)\right)}{-2 \kappa_{1}\left|\zeta-\kappa_{3}\right|^{2}} \sim \frac{s \kappa_{3}}{-2 r \kappa_{1}}+\frac{s-\kappa_{1}}{-2 \kappa_{1}}=\frac{c+1}{2},
\end{aligned}
$$

where $r$ is the radius of curvature in $\kappa_{3}$ :

$$
r=\lim \frac{\eta^{2}}{2\left(\xi-\kappa_{3}\right)}=\frac{-s \kappa_{3}}{s+\kappa_{1} c} .
$$

The impact parameter is then our dimensionless invariant $c$, up to a linear transformation. As to the scattering angle, it can be computed from an integral taken along the $\zeta$ orbit:

$$
\Delta \varphi_{1}=\left[\arg z_{1}\right]_{t=-\infty}^{+\infty}=\oint-s K \frac{\operatorname{Re}\left(\frac{\zeta^{2}+d \zeta}{\zeta^{2}+d \zeta-\kappa_{2} \kappa_{3}}\right)}{\operatorname{Re}\left(\frac{\zeta\left[\vec{\zeta}\left(\zeta^{2}+d \zeta-Q\right)-s K(\zeta+d)\right]}{\zeta^{2}+d \zeta-\kappa_{2} \kappa_{3}}\right)} \mathrm{d}(\arg \zeta)
$$

This integral can be carried out exactly for $c=0$, when the $\zeta$ orbit is a circle:

$$
\begin{aligned}
\Delta \varphi_{1} & =\int_{0}^{2 \pi} \frac{2 K+d}{2(K+d)}\left(1-\frac{K d}{2 K^{2}+2 K d+d^{2}+2 K(K+d) \cos \theta}\right) \mathrm{d} \theta \\
& = \begin{cases}\pi & \text { if }\left|\kappa_{1}\right|>|K| \\
\left(1-2 \frac{K}{\kappa_{1}}\right) \pi & \text { if }\left|\kappa_{1}\right|<|K| .\end{cases}
\end{aligned}
$$

The bifurcation set for this case is made, in the $\zeta$ plane, of the following lines: the set of collisions $\left(\infty, \kappa_{3},-\kappa_{2}\right)$, the boundary between the two regimes, the point $P_{1}$ and the orbit $c=c\left(P_{2}\right)$.

\section{The three particular $\vec{\kappa}$ points}

(a) Point A (strengths -1,1,1). Direct and exchange diffusion.

The $\zeta$ plane looks like the one Figure 3 assumed continuously deformed so as to admit the origin as a center of symmetry: $M_{3}=P_{1}$, symmetric of $M_{2}$, and $P_{2}=0$.

Aref (1979) has extensively studied the motion and we shall only briefly summarize it. The results obtained for $\kappa_{1}+\kappa_{2}=0$ still apply, but a new 
physical situation arises from the existence of $\zeta$ orbits which go from $\zeta=$ $\pm K$ to $\zeta=\mp K$ (these are all the orbits which cross the segment $T_{1} T_{2}$ ): the motion is then an exchange scattering in which the incident pair $\left(\begin{array}{ll}1 & 2\end{array}\right)$ is different from the outgoing doublet (1 3 ).

To sum up, three generic situations exist, depending on the initial conditions:

- $\left|\frac{\zeta}{K} \pm 1\right|>2$ : in uniformly rotating axes, the absolute motion is periodic.

- $\left|\frac{\zeta}{K} \pm 1\right|<2$ and $-8<c<1$ : exchange scattering; the integral giving the scattering angle is to be taken from 0 to $\pi$ only.

- $\left|\frac{\zeta}{K} \pm 1\right|<2$ and $c$ outside $[-8,1]$ : direct scattering as previously described.

The reduced forms of all the elliptic integrals giving the period and the scattering angle are gathered in Appendix I.

A particular case of exchange scattering is easy to solve and will give an idea of the motion:

For $c=0$, the $\zeta$ orbit is a half-circle and, computing $\frac{\mathrm{d}^{2} z_{1}}{\mathrm{~d} t^{2}}$ by deriving Equation (6), we find zero, which means a uniform linear motion $M_{1}$, hence a scattering angle of $\pi$. The motion of $\zeta=K e^{i \theta}$ is ruled by $\frac{\mathrm{d} \theta}{\mathrm{d} t}=\frac{v}{a} \sin ^{2} \theta$ which integrates as $\zeta=K \frac{i a-v t}{\sqrt{a^{2}+v^{2} t^{2}}}$ where we have noted $a=2 e^{2 \pi E / K^{2}}$, $v=\frac{K}{\pi a}$.

The origin of time being chosen when $M_{1}$ is the summit of an isosceles rectangle triangle, the absolute motions take place on three parallel straight lines:

$$
\left\{\begin{array}{l}
z_{1}=a+i v t \\
z_{2}=\frac{a+i v t}{2}+\frac{i}{2} \sqrt{a^{2}+v^{2} t^{2}} \\
z_{3}=\frac{a+i v t}{2}-\frac{i}{2} \sqrt{a^{2}+v^{2} t^{2}} .
\end{array}\right.
$$

The exchange scattering process is clearly seen on the above equations, and every other exchange scattering motion can be thought of as a continuous deformation of this one.

The bifurcation set is made of the set of collisions and of the boundaries of the domains limiting the three generic situations; note that the circle $J=0(|\zeta|=|K|)$ does not belong to it. This set is simple enough to be represented without any ambiguity by a $\mathrm{c}$ axis with the number and type of solutions in each interval: 


\begin{tabular}{|c|c|c|c|}
\hline \multicolumn{2}{|c|}{-8} & & \\
\hline $\begin{array}{l}2 \text { direct } \\
\text { scattering }\end{array}$ & $\begin{array}{l}2 \text { exchange } \\
\text { scattering }\end{array}$ & $\begin{array}{l}1 \text { biperiodic } \\
2 \text { exch. scatt. }\end{array}$ & $\begin{array}{l}2 \text { direct } \\
\text { scattering }\end{array}$ \\
\hline
\end{tabular}

(b) Point B summit of the quartic (strengths $-5,4,4$ ).

Nothing special happens to the motion. At the triple point $\zeta=0$, the vortex of strength $\frac{-5 K}{3}$ is motionless as it coincides with the barycentrum, and the two other vortices, which are symmetric with regard to the barycentrum, obey the circular motion. The $\zeta$ orbits around this stable point are equivalent to the quartics of equation

$$
2^{-4 / 3} c+1-\frac{15}{8}\left(\frac{\eta}{K}\right)^{2}-\frac{45}{256}\left(\frac{\xi}{K}\right)^{4}=0,
$$

and they correspond to ordinary motions; however the period, which still behaves like $J \int \frac{\mathrm{d} \xi}{\eta}$, diverges as $\left(c+2^{4 / 3}\right)^{-1 / 4}$.

(c) Point C (strengths $-2, \sqrt{3}, 2)$ conjunction of $\Delta=0$ and $\Pi\left(\kappa_{j}+\kappa_{\ell}\right)=0$. This point has no new properties: the previously studied singularities only add without interfering.

\section{Volume of the phase space}

The Hamiltonian system has an invariant element of volume of the phase space equal to

$$
\mathrm{d} v=\kappa_{1} \kappa_{2} \kappa_{3} \mathrm{~d} x_{1} \wedge \mathrm{d} y_{1} \wedge \mathrm{d} x_{2} \wedge \mathrm{d} y_{2} \wedge \mathrm{d} x_{3} \wedge \mathrm{d} y_{3} .
$$

Since there exist four real invariants $E, J, X, Y(X+i Y=B)$, we want the density of states $\frac{\mathrm{d} v}{\mathrm{~d} E \mathrm{~d} J \mathrm{~d} X \mathrm{~d} Y}$ after integration over two independent variables of the phase space.

By using the two successive changes of variables $\left(z_{1}, z_{2}, z_{3}\right) \rightarrow\left(z_{2}-\right.$ $\left.z_{3}, \zeta, B\right)$ and

$$
\left(\zeta, \bar{\zeta}, z_{2}-z_{3}, \overline{z_{2}-z_{3}}\right) \rightarrow\left(E, J, z_{2}-z_{3}, \overline{z_{2}-z_{3}}\right),
$$

whose jacobians are respectively

$$
\frac{D\left(z_{2}-z_{3}, \zeta, B\right)}{D\left(z_{1}, z_{2}, z_{3}\right)}=\frac{-s K}{z_{2}-z_{3}}
$$

and

$$
\frac{D(E, J)}{D(\zeta, \bar{\zeta})}=\frac{i \kappa_{1}^{2} \kappa_{2} \kappa_{3}}{2 \pi}\left|z_{2}-z_{3}\right|^{2} \frac{\operatorname{Im}\left(\zeta^{2}+d \zeta\right)}{\left|\left(\zeta-\kappa_{3}\right)\left(\zeta+\kappa_{2}\right)\right|^{2}}
$$


we obtain

$$
\begin{aligned}
\mathrm{d} v= & \frac{\pi}{\kappa_{1} s^{2} K^{2}} \frac{\left|\left(\zeta-\kappa_{3}\right)^{2}\left(\zeta+\kappa_{2}\right)^{2}\right|}{\operatorname{Im}\left(\zeta^{2}+d \zeta\right)} \\
& \mathrm{d}\left(x_{2}-x_{3}\right) \wedge \mathrm{d}\left(y_{2}-y_{3}\right) \wedge \mathrm{d} E \wedge \mathrm{d} J \wedge \mathrm{d} X \wedge \mathrm{d} Y,
\end{aligned}
$$

and we still have to integrate over $x_{2}-x_{3}$ and $y_{2}-y_{3}$. Since $\left|z_{2}-z_{3}\right|$ moves in time according to

$$
2 \pi \frac{\mathrm{d}\left|z_{2}-z_{3}\right|^{2}}{\mathrm{~d} t}=2 \kappa_{1} s^{2} \frac{\operatorname{Im}\left(\zeta^{2}+d \zeta\right)}{\left|\left(\zeta-\kappa_{3}\right)\left(\zeta+\kappa_{2}\right)\right|^{2}},
$$

the integration is quite easy to perform and we finally get

$$
\mathrm{d} v=\frac{\pi}{K^{2}} T(E, J) \mathrm{d} E \wedge \mathrm{d} J \wedge \mathrm{d} X \wedge \mathrm{d} Y,
$$

which shows that the density of states is the period of the reduced motion. This is a well known result of the theory of adiabatic invariants (see e.g. Landau and Lifchitz), for energy and time are conjugate variables: when $J$ is constant and $E$ slowly varying, then the product ET is constant since the volume of the phase space if conserved.

To take into account the fact that the phase space is the union of disconnected parts, we must sum the above expression over the number (between 0 and 4, see Table 1) of different domains associated to given values of $E$ and $J$. The resulting volume $\Omega(J, E)$ obeys the scaling law:

$$
\Omega(E, J)=\frac{\pi}{K^{2}} \sum_{\text {domains }} T(E, J)=\left|\frac{J}{Q K^{3}}\right| f\left(\frac{J}{Q} e^{4 \pi \frac{E}{Q}}\right) .
$$

Onsager (1949) defined the entropy $S$ and the temperature $\tau$ of an assembly of a large number of interacting vortices:

$$
S=k_{B} \log \Omega, \quad \frac{1}{\tau}=\frac{\mathrm{d} S}{\mathrm{~d} E} .
$$

Although it makes no sense to speak of thermodynamics about an integrable system, there may be some interest for the understanding of the behaviour of a large number of vortices to examine what the Onsager's theory gives when formally applied to the three-vortex system.

The main hypothesis made by Onsager is that the total amount of volume $\int_{-\infty}^{+\infty} \Omega \mathrm{d} E$ available to the system is finite, an hypothesis equivalent to assume the system confined in a box since the phase space and the configuration space are the same. Due to the scaling law for $\Omega$, the integral $\int \Omega \mathrm{d} E$ will be either finite and proportional to $J$ or infinite, depending on the strengths of the vortices. The first arising question is therefore: when 
$J$ is kept constant, is the integral $\int \Omega(E, J) \mathrm{d} E=\frac{Q}{4 \pi} \int_{c Q J>0} \Omega \frac{\mathrm{d} c}{c}$ finite or not, i.e. are all the singularities of $T$ integrable or not?

The singularities of $T$ are: the set of collisions, the unstable relative equilibria and, since $J$ is kept nonzero, the limit $e^{4 \pi E / Q} \rightarrow 0$ in the domains $\kappa_{1} \kappa_{2} \kappa_{3} K<0$ only.

(a) Two vortices close to each other (vortex $j$ alone): then

$$
T \sim \frac{4 \pi^{2} J}{\left(K-\kappa_{j}\right)^{2} \kappa_{j}}\left(\frac{Q c}{\kappa_{j}\left(K-\kappa_{j}\right)}\right)^{-\frac{Q \kappa_{j}}{\kappa_{1} \kappa_{2} \kappa_{3}}} \rightarrow 0,
$$

and the singularity $\int T \mathrm{~d} E=\frac{Q J}{4 \pi} \int \frac{T}{J} \frac{\mathrm{d} c}{c}$ is integrable.

(b) $\zeta$ tends to an unstable $\zeta_{0}$ value, whose $c$ value is $c_{o}$. The equivalent hyperbola having for equation:

$$
\begin{aligned}
G(\zeta, \bar{\zeta}) \equiv & \frac{c-c_{o}}{c_{o}}+\frac{J \kappa_{1} \kappa_{2} \kappa_{3}}{2 Q\left(\kappa_{1}\left|\zeta_{0}\right|^{2}+\kappa_{2} \kappa_{3} K\right)^{2}} \\
& \times\left[\mu_{o}\left(\zeta-\zeta_{o}\right)^{2}+2 v_{o}\left|\zeta-\zeta_{o}\right|^{2}+\bar{\mu}_{o}\left(\bar{\zeta}-\bar{\zeta}_{o}\right)^{2}\right]=0
\end{aligned}
$$

the period is equivalent to

$$
T \sim \operatorname{cst} J \int \frac{\mathrm{d} \bar{\zeta}}{G_{\zeta}^{\prime}(\zeta, \bar{\zeta})} \sim \operatorname{cst} J \log \left|c-c_{o}\right|,
$$

and the singularity is therefore integrable.

(c) $|\zeta|^{2} \rightarrow-\frac{\kappa_{2} \kappa_{3} K}{\kappa_{1}}$ (possible in every domain, except 0 and 150 ).

Since $c$ tends to zero with $J$ being kept constant and nonzero, then $e^{4 \pi E / Q}$ tends to zero, therefore the period $T=\operatorname{cst} e^{-4 \pi E / Q}$ has a nonintegrable singularity.

In conclusion, the volume of the phase space, with $J$ being constant, is finite and proportional to $J$ in the domains 0,150 and $K=0$ and infinite elsewhere. The unit of time we chose, i.e. $4 \pi^{2} \frac{J}{Q K}$, is a posteriori convenient for it is proportional to the volume.

Let us now examine the behaviour of the thermodynamical quantities, keeping in mind that any conclusion is meaningless for three vortices and can only be indicative for a larger system. For instance in the domain 0 (i.e. $\kappa_{j} K>0$ ), the function $E_{o} \rightarrow \int_{E<E_{o}} \Omega \mathrm{d} E$ is of course increasing, but the integrand $\Omega$, which is zero at the edges $E \rightarrow \pm \infty$, positive and integrable, has three infinite maxima at finite values of the energy (Figure 7). Each of these maxima corresponds to an unstable relative equilibrium configuration or, in other words, to a point of the phase space which links two disconnected domains. This feature somehow complicates the correspondence 
between energy and temperature and there exists some numerical evidence (Lundgren and Pointin, 1977, and references herein) of a possible lack of ergodicity which could come out of a multiple connexity of the phase space. Another interesting observation is that, when the energy tends to $+\infty$, the "temperature" of the three vortex system tends to a constant, negative value (Fig. 7), a fact already noticed for a large number of vortices by Lundgren and Pointin (page 334), C.E. Seyler (1974), Edwards and Taylor (1974, page 262).

\section{Conclusion}

In addition to the fact of being an exactly soluble three body problem, the three vortex system is very interesting in connection with the theory of turbulence. Unfortunately its number of degrees of freedom is too small to yield a chaotic behaviour (the threshold for such a behaviour is 3 ) and this was confirmed by the results: nonperiodic behaviours are obtained only for very particular values of the parameters. A four vortex system (see some preliminary results in Conte, 1979), with its 3 independent degrees of freedom and because we do not know about its integrability, is the really interesting dynamical system to study in order to have some hints about the integrability of the $\mathrm{N}$ vortex system.

\section{Acknowledgements}

We want to thank Y. Pomeau for many fruitful discussions which led to the discovery of the appropriate plane. We also greatly appreciated the formal Reduce-like computer language AMP (Drouffe, 1976) which helped us to establish the numerous necessary formulae.

The work is the first chapter of the unpublished Thèse d'État of the first author. It is an honor and a pleasure for us to dedicate it to Professor Hao Bailin and to wish him a long life.

\section{References}

H. Aref (1979), Motion of three vortices, Phys. Fluids 22, 393-400.

Bateman manuscript project, (1953), Higher transcendental functions, vol. II, chapter XIII; A. Erdélyi Editor, Mc Graw Hill.

R. Conte (1979), Thèse d'Etat, Université de Paris VI.

J.M. Drouffe, AMP language, (1976), same address as authors. 
S.F. Edwards and J.B. Taylor (1974), Negative temperature states of twodimensional plasmas and vortex fluids, Proc. Roy. Soc. London, A 336, $257-271$.

W. Gröbner and Hofreiter (1965), Integraltafel, vol. 4, Springer-Verlag.

L. Landau and E. Lifchitz (1960), Mechanics, Pergamon Press.

T.S. Lundgren and Y.B. Pointin (1977), Statistical mechanics of two-dimensional vortices, Journal of statistical physics, 17, 323-325.

A.M. Mayer (1878), Floating magnets, Nature, 18, 258.

E.A. Novikov (1975), Dynamics and statistics of a system of vortices, JETP 41, 937-943.

L. Onsager (1949), Statistical hydrodynamics, Nuovo Cimento, 6 suppl., 279-287.

H. Poincaré (1893), Théorie des tourbillons, pages 77-84, Deslis frères, Paris.

C.E. Seyler, Jr. (1974), Partition function for a two-dimensional plasma in the random phase approximation, Phys. Rev. Letters 32, 515-517.

S. Smale (1970), Topology and mechanics, Inventiones math., 10, 305-331 and $11,45-64$.

W. Thomson (1878), Floating magnets (illustrating vortex-systems), Nature, $18,13-14$. 


\section{Appendix I}

\section{Cases of integrability}

For practical applications, it may be of interest to find which values of the $\kappa_{j}$ 's lead to integrable expressions for the period (12). A first case is when two strengths are equal: $\kappa_{2}=\kappa_{3}$; then $\xi \eta$ can be expressed only with $|\zeta|^{2}$, using (11), and the period is a simple integral in the variable $|\zeta|^{2}$, which can be easily integrated numerically.

Another case is when, the strengths being rational, the algebraic curve (11) is of genus one or zero (the genus of an algebraic curve of degree $n$ is equal to $\frac{(n-1)(n-2)}{2}$ minus the number of double points). The only curve of genus zero is the circle $J=0$ but then the period is given by another nonintegrable expression. If the trajectory is of genus one, the abelian integral expressing the period can always be reduced to an elliptic integral by a birational transformation of the coordinates (see e.g. Bateman 1953). For small integer values of the strengths, there is some chance of finding curves of genus one.

Let us just mention three particular cases.

$\vec{k}=(1,1,1) K / 3$. This belongs to the first but not to the second case (degree 6 , genus 4 in general). The period is expressed by the hyperelliptic integral in $u=\frac{|\zeta|^{2}}{\kappa_{1}^{2}}$ :

$$
\frac{T}{T_{u}}=\frac{1}{2 \pi} \oint \frac{-9(u+3) \operatorname{sign}(\xi \eta)}{\sqrt{27 c^{3}(u+1)^{2}-2(u+3)^{3}} \sqrt{2(u+3)^{3}-27 c^{3}(u-1)^{2}}} \mathrm{~d} u .
$$

Novikov gave this expression in the variable $b=\frac{6}{u+3}=\frac{3 \kappa_{1}^{2}\left|z_{2}-z_{3}\right|^{2}}{J}$ which always remains between 0 and 2 but he did not integrate it:

$$
\frac{T}{T_{u}}=\frac{3}{4 \pi c^{3}} \oint \frac{\operatorname{sign}(\xi \eta)}{\sqrt{f(b)} \sqrt{-g(b)}} \mathrm{d} b
$$

with $f(b) \equiv b(b-3)^{2}-\frac{4}{c^{3}}, g(b) \equiv b\left(b-\frac{3}{2}\right)^{2}-\frac{1}{c^{3}}$. This hyperelliptic integral happens to be reducible to an elliptic integral (Bolza, 1898, mentioned in the tables of Gröbner and Hofreiter, 1965) of the variable $z=\frac{g(b)}{3 b}$, due to the relations:

$$
\begin{gathered}
\varphi(z) \equiv z^{3}-\frac{3}{2} z^{2}+\left(\frac{9}{16}-\frac{3}{2 c^{3}}\right) z+\frac{1}{8 c^{3}}-\frac{1}{4 c^{6}}=\frac{f(b)[h(b)]^{2}}{27 b^{3}}, \\
\frac{\mathrm{d} z}{\mathrm{~d} b}=\frac{6 h(b)}{9 b^{2}}
\end{gathered}
$$


with $h(b) \equiv b^{3}-\frac{3}{2} b^{2}+\frac{1}{2 c^{3}} ;$ this gives for the period:

$$
\frac{T}{T_{u}}=\frac{1}{8 \pi c^{3}} \oint \frac{\operatorname{sign}(\xi \eta) \operatorname{sign}(h(b))}{\sqrt{-z \varphi(z)}} \mathrm{d} z
$$

Let us call $b_{1}<b_{2}<b_{3}$ the zeros of $f, b_{4}<b_{5}<b_{6}$ those of $g$ ( $b_{4}$ and $b_{5}$ are not real for $\left.1<c^{3}<2\right), b_{7}<b_{8}<b_{9}$ those of $h$ and $z_{1}<z_{2}<z_{3}$ those of $\varphi$. The correspondence is $\left(b_{1}, b_{9}\right) \rightarrow z_{1},\left(b_{2}, b_{8}\right) \rightarrow z_{2},\left(b_{3}, b_{7}\right) \rightarrow z_{3}$, which gives the following values of the period for the two domains:

$$
\begin{gathered}
1<c^{3}<2: \operatorname{sign}(K) \oint=2 \int_{b_{1}}^{b_{2}} \mathrm{~d} b=6 \int_{z_{1}}^{z_{2}} \mathrm{~d} z, \\
b_{7}<b_{1}<b_{8}<b_{9}<b_{2}<b_{6}<b_{3} \\
\frac{T}{\left|T_{u}\right|}=\frac{3}{2 \pi c^{3}} \frac{K(k)}{\sqrt{\left(z_{3}-z_{2}\right)\left(-z_{1}\right)}}, \quad k^{2}=\frac{z_{3}\left(z_{2}-z_{1}\right)}{\left(z_{3}-z_{2}\right)\left(-z_{1}\right)}, \quad z_{1}<z_{2}<0<z_{3} \\
2<c^{3}: b_{7}<b_{1}<b_{4}<b_{8}<b_{5}<b_{9}<b_{6}<b_{2}<b_{3}, \quad z_{1}<0<z_{2}<z_{3} .
\end{gathered}
$$

Two equivalent expressions lead to the period, according to whether $\zeta$ turns around $M_{1}$ or another vortex:

$$
\begin{gathered}
\left(\zeta \text { around } M_{1}\right) \quad: \operatorname{sign}(K) \oint=4 \int_{b_{1}}^{b_{4}} \mathrm{~d} b=4 \int_{z_{1}}^{o} \mathrm{~d} z \\
\left(\zeta \text { around } M_{2} \text { or } M_{3}\right): \operatorname{sign}(K) \oint=2 \int_{b_{5}}^{b_{6}} \mathrm{~d} b=4 \int_{z_{1}}^{o} \mathrm{~d} z \\
\quad \frac{T}{\left|T_{u}\right|}=\frac{K(k)}{\pi c^{3} \sqrt{z_{3}\left(z_{2}-z_{1}\right)}}, \quad k^{2}=\frac{-z_{1}\left(z_{3}-z_{2}\right)}{z_{3}\left(z_{2}-z_{1}\right)}
\end{gathered}
$$

$\vec{k}=(-1,1,1)$,$K where three generic situations exist. We assume K>0$.

The $\zeta$ curves are bicircular quartics of genus one and we derive below the normal forms of the scattering angle and the period of the reduced motion:

$$
\begin{aligned}
\Delta \varphi_{1} & =\oint \frac{\left(u+u_{o}\right) \operatorname{sign}(\xi \eta)}{2 u \sqrt{\left(u-u_{-}\right)\left(u-u_{+}\right)} \sqrt{(u-1)\left(u_{o}-u\right)}} \mathrm{d} u \\
\frac{T}{T_{u}} & =\frac{1}{2 \pi} \oint \frac{4 \operatorname{sign}(\xi \eta)}{c(u-1) \sqrt{\left(u-u_{-}\right)\left(u-u_{+}\right)} \sqrt{(u-1)\left(u_{o}-u\right)}} \mathrm{d} u
\end{aligned}
$$

with the notations $u=\left|\frac{\zeta}{K}\right|^{2}, u_{o}=1+\frac{8}{c}, u_{ \pm}=\frac{4}{c}-1 \pm \frac{4}{c} \sqrt{1-c}$. The variable $b$ in Aref is related to $u$ by $b=\frac{6}{1-u} . K, E$ and $\Pi$ are the complete elliptic 
integrals of the first, second and third kind, the last one being defined a:*

$$
\Pi(n, k)=\int_{0}^{1} \frac{\mathrm{d} x}{\left(1-n x^{2}\right) \sqrt{\left(1-x^{2}\right)\left(1-k^{2} x^{2}\right)}}
$$

First regime (exchange scattering). $-8<c<1 . \oint \operatorname{sign}(\xi \eta) \mathrm{d} u=2 \int_{1}^{u_{-}} \mathrm{d} u$.

There is no discontinuity for $c=0$ where $\Delta \varphi_{1}$ evaluates to $\pi$.

$$
\begin{gathered}
-8<c<0: \Delta \varphi_{1}=\frac{-2}{\sqrt{\left(1-u_{o}\right)\left(u_{-}-u_{+}\right)}} \\
\quad\left[\left(1+\frac{u_{0}}{u_{+}}\right) K(k)+\frac{u_{o}\left(u_{+}-1\right)}{u_{+}} \Pi(n, k)\right] \\
\text { with } k^{2}=\frac{\left(1-u_{-}\right)\left(u_{o}-u_{+}\right)}{\left(1-u_{o}\right)\left(u_{-}-u_{+}\right)}, \quad n=\frac{u_{+}\left(u_{-}-1\right)}{u_{-}-u_{+}}, \\
0<c<1: \Delta \varphi_{1}=\frac{2}{\sqrt{\left(u_{o}-u_{-}\right)\left(u_{+}-1\right)}}\left[2 K(k)+\frac{8}{c} \Pi(n, k)\right] \\
\text { with } k^{2}=\frac{\left(u_{o}-u_{+}\right)\left(u_{-}-1\right)}{\left(u_{o}-u_{-}\right)\left(u_{+}-1\right)}, \quad n=\frac{u_{o}\left(1-u_{-}\right)}{u_{o}-u_{-}}
\end{gathered}
$$

Second regime (direct scattering). $c<-8$ or $1<c . \oint \operatorname{sign}(\xi \eta) \mathrm{d} u=2 \int_{1}^{u_{o}} \mathrm{~d} u$

$$
\begin{aligned}
c<-8: \Delta \varphi_{1} & =\frac{-2}{\sqrt{\left(1-u_{-}\right)\left(u_{o}-u_{+}\right)}} \\
& {\left[\left(1+\frac{u_{0}}{u_{+}}\right) K(k)+\frac{\left(u_{+}-1\right) u_{o}}{u_{+}} \Pi(n, k)\right] } \\
\text { with } k^{2}= & \frac{\left(1-u_{o}\right)\left(u_{-}-u_{+}\right)}{\left(1-u_{-}\right)\left(u_{o}-u_{+}\right)}, \quad n=\frac{u_{+}\left(u_{o}-1\right)}{u_{o}-u_{+}}, \\
1<c: \Delta \varphi_{1}= & \left(u_{o}^{-\frac{1}{4}}-u_{o}^{\frac{1}{4}}\right) K(k)+\frac{\left(1+\sqrt{u_{o}}\right)^{2}}{2} \Pi(n, k) \\
\text { with } k^{2}= & \frac{\left(u_{o}-1\right)^{2}-4\left(\sqrt{u_{o}}-1\right)^{2}}{16 \sqrt{u_{o}}}, \quad n=-\frac{\left(1-\sqrt{u_{o}}\right)^{2}}{4 \sqrt{u_{o}}}
\end{aligned}
$$

\footnotetext{
${ }^{*}$ In the tables of Gradshteyn and Ryzhik $4^{\text {th }}$ edition, the definition 8.111.4 is not consistent with the rest of the book; many formulae concerning elliptic integrals are wrong, among them 3.132.5, 3.132.6, 3.138.8, 3.148.1.
} 
Third regime (biperiodic). $0<c<1 \oint \operatorname{sign}(\xi \eta) \mathrm{d} u=4 \int_{u_{+}}^{u_{o}} \mathrm{~d} u$

$$
\begin{gathered}
\frac{T}{T_{u}}=\frac{2}{\pi} \sqrt{\frac{2\left(u_{o}-1\right)}{\left(u_{-}-1\right)\left(u_{o}-u_{-}\right)}}\left[K(k)-\frac{u_{o}-u_{-}}{6\left(u_{o}-1\right)} E(k)\right] \\
\Delta \varphi_{1}=\frac{4}{\sqrt{\left(u_{-}-1\right)\left(u_{o}-u_{-}\right)}}\left[2 K(k)-\frac{8}{c+8} \Pi(n, k)\right] \\
\text { with } k^{2}=\frac{\left(u_{-}-1\right)\left(u_{o}-u_{+}\right)}{\left(u_{+}-1\right)\left(u_{o}-u_{-}\right)}, \quad n=\frac{u_{+}-u_{o}}{u_{o}\left(u_{+}-1\right)}
\end{gathered}
$$

$\vec{\kappa}=(-1,2,2) \frac{K}{3}$, a case with two possible regimes (biperiodic, expanding).

The $\zeta$ trajectories are the Cassini ovals, whose genus is one. The period for instance is given by

$$
T=\left(\frac{4 \pi^{2} J}{K^{3}}\right) \frac{\alpha}{2 \pi} \oint \frac{-27 \operatorname{sign}(\xi \eta)}{4(u-3)^{2} \sqrt{(u+1)^{2}-\alpha} \sqrt{\alpha-(u-1)^{2}}} \mathrm{~d} u
$$

with $u=\left|\frac{\zeta}{\kappa_{2}}\right|^{2}, \alpha=\left|\left(\frac{\zeta}{\kappa_{2}}\right)^{2}-1\right|^{2}=16 e^{18 \pi E / K^{2}}$. Its reduced form is not very compact and we shall not give it here.

\section{Appendix II}

\section{Stability of the relative equilibria}

In order to obtain the shape of the $\zeta$ trajectories in the vicinity of the relative equilibria we must determine whether they are of elliptic or hyperbolic nature.

The points $\infty,-\kappa_{2}, \kappa_{3}$ are elliptic, neighbouring orbits are circles described with a uniform circular motion of period:

$$
T=\frac{4 \pi^{2} J}{\left(K-\kappa_{j}\right)^{2} \kappa_{j}}\left(\frac{Q c}{\kappa_{j}\left(K-\kappa_{j}\right)}\right)^{-\frac{Q \kappa_{j}}{\kappa_{1} \kappa_{2} \kappa_{3}}}
$$

We now assume that $\zeta_{o}$ is the affix of an ordinary relative equilibrium (the case of two coincident r.e. is studied elsewhere in the paper), which implies $J \neq 0$ and we study the vicinity of the equilateral triangles and of the aligned configurations. 
By writing $f(\zeta, \bar{\zeta})$ for the right-hand side of Equation (10), the small motions of a $\zeta$ point in the vicinity of a stationary point $\zeta_{o}$ are ruled by:

$$
2 \pi i \frac{\mathrm{d} \bar{\zeta}}{\mathrm{d} t}=\left(\zeta-\zeta_{o}\right) \frac{\partial f}{\partial \zeta}\left(\zeta_{o}, \bar{\zeta}_{o}\right)+\left(\overline{\zeta-\zeta_{o}}\right) \frac{\partial f}{\partial \bar{\zeta}}\left(\zeta_{o}, \bar{\zeta}_{o}\right),
$$

or, in real matricial notation:

$$
2 \pi \frac{\mathrm{d}}{\mathrm{d} t}\left(\begin{array}{l}
\xi \\
\eta
\end{array}\right)=M\left(\begin{array}{l}
\xi-\xi_{o} \\
\eta-\eta_{o}
\end{array}\right)=\left(\begin{array}{cc}
\alpha^{\prime}+\beta^{\prime} & \alpha-\beta \\
\alpha+\beta & -\alpha^{\prime}+\beta^{\prime}
\end{array}\right)\left(\begin{array}{l}
\xi-\xi_{o} \\
\eta-\eta_{o}
\end{array}\right)
$$

with $\alpha+i \alpha^{\prime}=\frac{\partial f}{\partial \zeta}\left(\zeta_{o}, \bar{\zeta}_{o}\right)=\mu, \beta+i \beta^{\prime}=\frac{\partial f}{\partial \zeta}\left(\zeta_{o}, \bar{\zeta}_{o}\right)=\nu$.

The stability condition is: $\operatorname{tr}(M)=0, \operatorname{det}(M)>0$. We find:

$$
\frac{-\left(\kappa_{1}|\zeta|^{2}+\kappa_{2} \kappa_{3} K\right)}{J\left(\zeta+\kappa_{2}\right)\left(\zeta-\kappa_{3}\right)}=\frac{\mu}{2|\zeta|^{2}+d \bar{\zeta}-s K}=\frac{\nu}{\zeta^{2}+d \zeta-Q}
$$

with the condition (17). The trace of $M$ is therefore zero. If $\zeta_{o}$ is elliptic, then the small motions have the period $4 \pi^{2} / \sqrt{\operatorname{det}(M)}$. We now divide the study according to the two types of stationary points.

(a) The equilateral triangles

$\operatorname{det}(M)=\frac{3 Q^{3}}{J^{2}}$. The stability condition is $Q>0$ and, when this is fulfilled, the period of the small motions is $\frac{4 \pi^{2} J}{\sqrt{3} Q^{3 / 2}}$; comparing with the period of the absolute motion which will be derived later, we find:

$$
\left(\frac{T_{r}}{T_{a}}\right)^{2}=1-\frac{1}{2 K^{2}} \sum_{j} \sum_{\ell>j}\left(\kappa_{j}-\kappa_{\ell}\right)^{2} .
$$

The overall rotation is therefore quicker than the small motions with equality only for identical strengths.

(b) The aligned configurations

$$
\operatorname{det} M=|\nu|^{2}-|\mu|^{2}=\left(\frac{\kappa_{1} \zeta^{2}+\kappa_{2} \kappa_{3} K}{J\left(\zeta^{2}+d \zeta-\kappa_{2} \kappa_{3}\right)}\right)^{2} F_{1}(\zeta) F_{2}(\zeta)
$$

with the notation

$$
\begin{aligned}
& F_{1}(\zeta)=-\zeta^{2}+K s-Q \\
& F_{2}(\zeta)=3 \zeta^{2}+2 d \zeta-s K-Q=P^{\prime}(\zeta)
\end{aligned}
$$

and the condition: $\zeta$ is a real zero of $P$. 
The determinant of $M$ changes sign when the resultant of $P$ and $F_{1} F_{2}$ vanishes. We find:

$$
\operatorname{res}\left(P, F_{1}\right)=-3 s^{2} Q^{2}, \quad \operatorname{res}\left(P, F_{2}\right)=s^{2} \Delta .
$$

Then we obtain the nature of the aligned configurations in the parameter space:

$\Delta>0(Q<0)$ : one stable configuration

$\Delta<0$ and $Q<0$ : two stable configurations, one unstable $\Delta<0$ and $Q>0$ : three unstable configurations.

It is worth observing that in the present problem the nonlinear stability is the same as the linear one. 